TRANSACTIONS OF THE

AMERICAN MATHEMATICAL SOCIETY

Volume 364, Number 8, August 2012, Pages 4239-4265

S 0002-9947(2012)05502-4

Article electronically published on March 13, 2012

\title{
TWO CRITICAL PERIODS IN THE EVOLUTION OF RANDOM PLANAR GRAPHS
}

\author{
MIHYUN KANG AND TOMASZ ŁUCZAK
}

\begin{abstract}
Let $P(n, M)$ be a graph chosen uniformly at random from the family of all labeled planar graphs with $n$ vertices and $M$ edges. In this paper we study the component structure of $P(n, M)$. Combining counting arguments with analytic techniques, we show that there are two critical periods in the evolution of $P(n, M)$. The first one, of width $\Theta\left(n^{2 / 3}\right)$, is analogous to the phase transition observed in the standard random graph models and takes place for $M=n / 2+O\left(n^{2 / 3}\right)$, when the largest complex component is formed. Then, for $M=n+O\left(n^{3 / 5}\right)$, when the complex components cover nearly all vertices, the second critical period of width $n^{3 / 5}$ occurs. Starting from that moment increasing of $M$ mostly affects the density of the complex components, not its size.
\end{abstract}

\section{INTRODUCTION}

Since the seminal work of Tutte 33 maps and graphs on 2-dimensional surfaces have become widely studied combinatorial objects in discrete mathematics. The enumerative and structural problems around maps, i.e. embedded graphs on a surface, are relatively well settled. Starting from the number of planar maps computed by Tutte 34, 33, the number of rooted maps on surfaces was found by Bender, Canfield, and Richmond 2], and other classes of maps were extensively enumerated since then. Such enumeration results have been used to study typical properties of random maps on surfaces, e.g. the size of the largest components by Banderier et al. [1.

Another important aspect of maps is that they allow nice bijections to the socalled well-labeled trees. The bijection between planar maps and the well-labeled trees was first studied amongst others by Schaeffer [31, which was extended by Bouttier, Di Francesco, and Guitter [6] to maps on an orientable surface with positive genus. These bijections are the cornerstone of profound results on the topological structure of scaling limits of random maps by Chassaing and Schaeffer [9], Le Gall [13, and Schramm [32.

On the other hand, analogous problems on related graphs that are embeddable on a surface are still wide open. The enumerative properties of random planar graphs have attracted much attention since the work of McDiarmid, Steger, and Welsh [29], who studied random labeled planar graphs with a given number of vertices.

Let $\mathrm{pl}(n)$ be the number of labeled planar graphs on $n$ vertices. McDiarmid, Steger, and Welsh 29 showed amongst other results that the quantity $(\operatorname{pl}(n) / n !)^{1 / n}$

Received by the editors June 2, 2010 and, in revised form, November 5, 2010.

2010 Mathematics Subject Classification. Primary 05C10, 05C80; Secondary 05C30, 05A16.

(C)2012 American Mathematical Society

Reverts to public domain 28 years from publication 
converges to a limit $\gamma$ as $n \rightarrow \infty$, which is called the growth constant. An upper bound $\gamma \leq 37.3$, based on the triangulations and probabilistic methods, was obtained by Osthus, Prömel, and Taraz [30. A lower bound $\gamma \geq 26.1$ was given by Bender, Gao, and Wormald [3, who studied the number of labeled 2-connected planar graphs through the singularity analysis of generating functions arising from the decomposition of graphs along connectivity. Using a similar method Giménez and Noy [16] proved that $\operatorname{pl}(n) \sim c n^{-7 / 2} \gamma^{n} n$ !, where $c, \gamma>0$ are explicitly computable constants (e.g. $\gamma \sim 27.2$ ). As for the number $s_{g}(n)$ of graphs embeddable on a surface with positive genus, McDiarmid [27] showed that its growth constant is the same as that of planar graphs, while Chapuy et al. [8] found $s_{g}(n)$ for all orientable surfaces of positive genus $g$ proving that $s_{g}(n) \sim \alpha_{g} n^{5(g-1) / 2-1} \gamma^{n} n$ !, where $\alpha_{g}>0$ and $\gamma$ is the growth constant of the labeled planar graphs. McDiarmid and Reed 28, studied various typical properties of random graphs on surfaces, e.g. subgraph containment and maximum degree.

Frieze [12] asked about the asymptotic behavior of the number $\mathrm{pl}(n, M)$ of labeled planar graphs on $n$ vertices with $M$ edges. Note first that if $M \leq$ an for some $a<1 / 2$, then a typical graph with $n$ vertices and $M$ edges is planar, i.e. $\operatorname{pl}(n, M)=(1+o(1))\left(\begin{array}{c}n \\ 2 \\ M\end{array}\right)$ (cf. 25] or [20]). Gerke et al. 14] proved the existence of its growth constant, in the sense that for $\mathrm{pl}(n, a n)$ with $0 \leq a \leq 3$, the quantity $(\operatorname{pl}(n, a n) / n !)^{1 / n}$ converges to a limit $\gamma_{a}$ as $n \rightarrow \infty$. The asymptotic formula for $\operatorname{pl}(n, a n)$ was found by Giménez and Noy [16, who showed that for $1<a<3$, there are analytic constants $c_{a}, \gamma_{a}>0$ such that $\operatorname{pl}(n$, an $) \sim c_{a} n^{-4} \gamma_{a}^{n} n$ !. In this paper we deal with the case when $a \in[1 / 2,1)$. Note that for such $a$

$$
\operatorname{pl}(n, M) \leq\left(\begin{array}{c}
n \\
2 \\
M
\end{array}\right)=n^{(1+o(1)) M},
$$

i.e., $\gamma_{a}=0$, and a 'naive' generating function approach does not lead to the asymptotic formula for $\mathrm{pl}(n, M)$.

We use $\mathrm{pl}(n, M)$ to study the asymptotic behavior of the uniform random planar graph $P(n, M)$, by which we mean a graph chosen uniformly at random among all labeled planar graphs with $n$ vertices and $M$ edges. Thus, each of these graphs occurs as $P(n, M)$ with probability $1 / \mathrm{pl}(n, M)$. From the results in [14, 16] it follows that if $M / n$ is bounded away from both 1 and 3, then $P(n, M)$ has a wellordered structure, for instance, it has a large component of size $n-O(1)$, and all planar graphs of finite size appear as its subgraphs. Thus, it corresponds to late stages of the evolution of the standard uniform random graph $G(n, M)$, the graph chosen uniformly at random among all graphs with $n$ vertices and $M$ edges. Our goal is to study the typical size and structure of components in $P(n, M)$ in a more interesting range, when $M \leq n$. It turns out that, somewhat surprisingly, $P(n, M)$ exhibits two critical ranges, which occur at $M=n / 2+O\left(n^{2 / 3}\right)$ and $M=n+O\left(n^{3 / 5}\right)$.

The first critical period corresponds to a phase transition phenomenon observed in the plethora of different random graph models. Let us recall some results on one of the most widely used random graph models, $G(n, M)$. It follows from the papers of Erdős and Rényi [11, Bollobás [5, Łuczak [21, Łuczak, Pittel, and Wierman 25], Janson et al. [19], and Janson [18] (see also Janson, Łuczak, and Ruciński 20]) that the giant component (i.e. the unique largest component) suddenly emerges at $M=n / 2+O\left(n^{2 / 3}\right)$, and nowadays this spectacular phenomenon is well studied and 
understood. If $M=n / 2+s$ and $-n \ll s \ll-n^{2 / 3}$, then, a.a.s. (i.e. with probability tending to 1 as $n$ approaches $\infty) G(n, M)$ consists of isolated trees and unicyclic components, and the largest component is a tree of size $(1+o(1)) \frac{n^{2}}{2 s^{2}} \log \frac{|s|^{3}}{n^{2}}$. On the other hand, if $n^{2 / 3} \ll s \ll n$, then a.a.s. $G(n, M)$ contains exactly one component with more edges than vertices of size $(4+o(1)) s$, while all other components are of size $o\left(n^{2 / 3}\right)$. Furthermore, if $s \gg n^{2 / 3}$, then a.a.s. $G(n, M)$ contains a topological copy of $K_{3,3}$, and thus it is not planar, while, as we have mentioned, for $s \ll-n^{2 / 3}$, a.a.s. $G(n, M)$ consists of isolated trees and unicyclic components, so it is clearly planar.

Another random structure relevant to the behavior of $P(n, M)$ is the uniform random forest $F(n, M)$ (i.e. a forest chosen uniformly at random among all labeled forests with $n$ vertices and $M$ edges). Luczak and Pittel 24] found that although the giant component in $F(n, M)$ emerges at $M=n / 2+O\left(n^{2 / 3}\right)$, as for $G(n, M)$, the critical behavior of these two models is somewhat different. Let $M=n / 2+s$. If $s \ll-n^{2 / 3}$, then the structure of both $F(n, M)$ and $G(n, M)$ is similar. In particular, the size of the largest tree in $F(n, M)$ is a.a.s. $(1+o(1)) \frac{n^{2}}{2 s^{2}} \log \frac{|s|^{3}}{n^{2}}$. However in the supercritical phase, when $s \gg n^{2 / 3}$, the giant tree of $F(n, M)$ is a.a.s. of size $(2+o(1)) s$, which is roughly half of the size of the largest component of $G(n, M)$, while the second largest tree of $F(n, M)$ is of size $\Theta\left(n^{2 / 3}\right)$, which does not depend much on $s$ provided $s \ll n$, i.e. it is by far larger than the second largest component of $G(n, M)$ for $m=n / 2+s$, which is of size $\Theta\left(n^{2} / s^{2} \log \left(s^{3} / n^{2}\right)\right)$.

In this paper we show that as far as $M=n / 2+s$, where $s \gg n^{2 / 3}$ and $s / n$ is bounded away from $1 / 2$, the behavior of $P(n, M)$ is similar to that of $F(n, M)$. Namely, a.a.s. the size of the largest complex component is of the order $(2+o(1)) s$, while the second largest component has $\Theta\left(n^{2 / 3}\right)$ vertices. However, unlike in the case of $F(n, M)$ for which $M \leq n-1$, for $P(n, M)$ we have only $M \leq 3 n-6$, so the rate of growth of the size of complex components must change at some point. We prove that it occurs when $M=n+O\left(n^{3 / 5}\right)$. More precisely, if $M=n+t$ and $t \ll-n^{3 / 5}$, then the complex components of $P(n, M)$ have a.a.s. $n-(2+o(1))|t|$ vertices altogether, while for $n^{3 / 5} \ll t \ll n^{2 / 3}$ they contain $n-(\alpha+o(1))(n / t)^{3 / 2}$ vertices for some computable constant $\alpha>0$. Let us mention that the condition $t \ll n^{2 / 3}$ is a result of the proof method we have used and most likely can be replaced by $t \ll n$. Furthermore, our method can say quite a lot about the structure of the largest component very much in the spirit of Łuczak 22.

The rest of the paper is organized as follows. In the next section we describe the main idea of our argument. Then, in Section 3, we present the first analytic ingredient of the proof: counting specially weighted cubic planar multigraphs using generating functions. Here we also describe how to use this result to bound the number of planar multigraphs with minimum degree three. Then, in the next section, we estimate the number of planar graphs with $k$ vertices and $k+\ell$ edges in which each component has more edges than vertices. Finally, in the main chapter of this paper, we use a direct counting to study the number $\mathrm{pl}(n, M)$ and the asymptotic properties of $P(n, M)$ for different values of $M$.

\section{IDEA OF THE PROOF}

As we have already mentioned most of results concerning the asymptotic behavior of $\operatorname{pl}(n, M)$ is based on the generating function method. Thus, for $a \in(0,3)$, 
one can study the function

$$
f_{a}(x)=\sum_{n} \frac{\operatorname{pl}(n, a n)}{n !} x^{n}
$$

and deduce the asymptotic behavior of $\operatorname{pl}(n, a n)$ from the behavior of $f_{a}(x)$ near its singularities. Note however that since

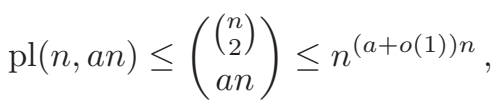

for $a<1$, the coefficients in the expansion of $f_{a}(x)$ tend to zero too fast to be handled by standard methods of generating function analysis. On the other hand, the condition that a graph is planar is very hard to grasp by purely combinatorial means. Thus, in this paper we use a combination of analytic and combinatorial tools. From a planar graph we extract its kernel, which is its only part responsible for the planarity and is dense enough to be treated by the generating functions method. Then we use a technically involved but rather natural counting argument to find asymptotic properties of $P(n, M)$.

In order to make the above description precise, we introduce some definitions. The excess $\operatorname{ex}(G)$ of a graph $G$ is the difference between the number of its edges and the number of its vertices. We call components of a graph with positive excess, i.e. those which have at least two cycles, complex components, and we say that the graph is complex if all its components are complex. The core of a graph $G$, denoted core $(G)$, is the maximal subgraph of $G$ with the minimum degree two. The kernel of $G$, denoted by $\operatorname{ker}(G)$, is obtained from the core by removing all isolated cycles and replacing each path whose internal vertices are all of degree two by an edge. Note that for a graph $G$ the kernel is a multigraph which can have multiple edges and loops. However, the excess of both $\operatorname{core}(G)$ and $\operatorname{ker}(G)$ are the same. Note also that $\operatorname{ker}(G)$ clearly has the minimum degree three. If $\operatorname{ker}(G)$ is cubic, we say that $G$ is clean. We define the deficiency $\operatorname{df}(G)$ of $G$ as the sum of degrees of vertices of $\operatorname{ker}(G)$ minus three times the number of vertices of $\operatorname{ker}(G)$. Therefore, a graph is clean if and only if its deficiency is zero.

Our argument is based on a simple observation that $G$ is planar if and only if $\operatorname{ker}(G)$ is planar. We use analytic methods to count the number of possible candidates for the kernel and then follow a purely combinatorial argument. To this end, in Section 3 we apply the singularity analysis to extract the asymptotic number of possible clean kernels of the planar graphs (Theorem 11). The proof is similar to that used in the case of counting cubic planar graphs presented in Bodirsky et al. 4, but in order to find the number of all planar graphs, we need to count cubic planar multigraphs with some special weight function dependent on the number of loops and multiple edges of a multigraph. Then, we use a simple combinatorial idea to generalize our estimates to the number of kernels with non-zero deficiency.

In the remaining part of our argument we follow an idea from Luczak 22. (see also Janson, Łuczak, and Ruciński [20]) and construct complex planar graphs $G$ from their kernels. Thus, we first choose the kernel, then put on its edges vertices of degree two obtaining the core of the graph, and add to it a forest rooted on vertices of the core. This procedure led to the estimate given in Theorem 2 , We also remark that such a typical complex planar graph on $k$ vertices consists of large components and, perhaps, some small components of combined excess $O(1)$. 
Finally, in the main part of the paper we count the number of planar graphs by splitting it into a complex planar graph, the number of which we have just found, and the part which consists of isolated trees and unicyclic components, whose number is well known (see Britikov [7]). We compute $\mathrm{pl}(n, M)$ for different values of $M=M(n) \leq n+o(n)$. At the same time, we get information on the typical structure of $P(n, M)$ such as the size of the largest component, its excess, and core.

\section{Cubic planar graphs}

In this section we study the family of cubic planar weighted multigraphs, which plays a crucial role in studying the kernel of a random planar graph. We then consider the family of 'supercubic' planar graphs that are planar weighted multigraphs with minimum degree at least three and a positive deficiency, which is indeed the set of possible kernels of complex planar graphs.

3.1. Cubic planar weighted multigraphs. In this section we count the number of all labeled cubic planar weighted multigraphs, where each multigraph with $f_{1}$ loops, $f_{2}$ double edges, and $f_{3}$ triple edges gets weight $2^{-f_{1}-f_{2}} 6^{-f_{3}}$. For $k=0,1$, let $g_{n}^{(k)}$ be the number of all labeled $k$-vertex connected cubic planar weighted multigraphs on $n$ vertices and let $G^{(k)}(x)$ be the corresponding exponential generating function defined by

$$
G^{(k)}(x):=\sum_{n \geq 0} \frac{g_{n}^{(k)}}{n !} x^{n} .
$$

Note that $g_{n}^{(k)}=0$ for odd $n$ and also for $n=0$, except that we set $g_{0}^{(0)}=1$ by convention. It is well known (e.g. [16, 17]) that

$$
G^{(0)}(x)=\exp \left(G^{(1)}(x)\right)
$$

The function $G^{(1)}(x)$ is defined by the following system of equations:

$$
\begin{aligned}
3 x \frac{d G^{(1)}(x)}{d x} & =D(x)+S(x)+\left(P(x)-x^{2} / 4+x^{2} / 12\right)+H(x) \\
& +\left(B(x)-x^{2} / 4+x^{2} / 8\right) \\
& =D(x)+C(x)-7 x^{2} / 24 \\
B(x) & =x^{2}(D(x)+C(x)) / 2+x^{2} / 4 \\
C(x) & =S(x)+P(x)+H(x)+B(x) \\
D(x) & =B(x)^{2} / x^{2}-x^{2} / 16 \\
S(x) & =C(x)^{2}-C(x) S(x) \\
P(x) & =x^{2} C(x)+x^{2} C(x)^{2} / 2+x^{2} / 4 \\
2(C(x)+1) H(x) & =u(1-2 u)-u(1-u)^{3} \\
x^{2}(C(x)+1)^{3} & =u(1-u)^{3} .
\end{aligned}
$$

This system of equations is obtained by following the lines of Sections 3-6 in Bodirsky et al. 4, where cubic planar simple graphs were studied, so below we just outline the argument.

The starting idea is that given a connected cubic planar weighted multigraph $G$, we select an arbitrary edge $e$ in $G$ and orient the edge $e$ to obtain a rooted 
counterpart $\hat{G}$. More precisely, the rooted cubic graph $\hat{G}=(V, E, s t)$ obtained from a connected cubic multigraph $G=(V, E)$ consists of $G=(V, E)$ and an ordered pair of adjacent vertices $s$ and $t$. The oriented edge $s t$ is called the root of $\hat{G}$. Denote by $G^{-}$a graph obtained from $\hat{G}$ by deleting the root of $\hat{G}$. We have the following lemma analogous to Lemma 1 in 4 .

Lemma 1. A rooted cubic graph $\hat{G}=(V, E$, st $)$ has exactly one of the following types:

- $b$ : the root is a self-loop.

- $d: G^{-}$is disconnected.

- $s: G^{-}$is connected, but there is a cut edge in $G^{-}$that separates $s$ and $t$.

- $p: G^{-}$is connected, there is no cut edge in $G^{-}$separating $s$ and $t$, and either st is an edge of $G^{-}$or $G \backslash\{s, t\}$ is disconnected.

- $h: G^{-}$is connected, there is no cut-edge in $G^{-}$separating s and $t, G$ is simple, and $G \backslash\{s, t\}$ is connected.
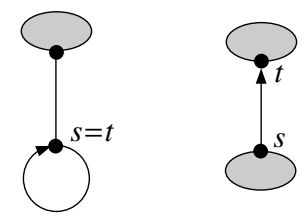

$b$-graph

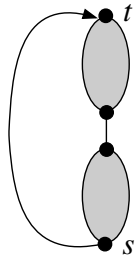

$s$-graph

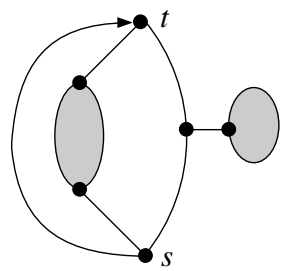

p-graph

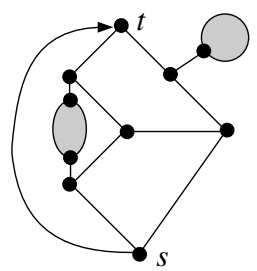

$h$-graph

Figure 1. The five types of rooted cubic graphs in Lemma 1

The generating function for the family $\mathcal{B}$ of $b$-graphs is denoted by $B(x)$. The other generating functions in (11) are analogously defined according to their corresponding types. Furthermore, the above system of equations follows from decomposition of graphs.

Note that the number of labeled connected cubic planar weighted multigraphs with one distinguished oriented edge is counted by $3 x \frac{d G^{(1)}(x)}{d x}$.

The difference between the system of equations above and that in Bodirsky et al. 4, arises as follows. In [4 the term $\left(B(x)-x^{2} / 4+x^{2} / 8\right)$ does not appear in $3 x \frac{d G^{(1)}(x)}{d x}$. The reason is that each graph in $\mathcal{B}$ enumerated by $B(x)$ works merely as a building block in $\hat{G}$ when considering simple graphs, while it may also appear as a connected component (whose root edge is a loop) when considering multigraphs. Note however that when considering weights, a graph on 2 vertices in $\mathcal{B}$ (that is, a rooted "dumbbell" consisting of two vertices, an edge, and two loops, one of which is marked as a root) gets a weight $1 / 2$ when it is used as a building block (one loop disappears in the building operation), but $1 / 4$ when used as an isolated component (due to two loops). In a similar way, a graph on 2 vertices in the family $\mathcal{P}$ enumerated by $P(x)$ (which is indeed a triple edge, one of which is oriented) gets a weight $1 / 2$ when it is used as a building block, but $1 / 6$ when used as an isolated component.

Analogous to Section 6 in 4, one can use the singularity analysis to obtain the following asymptotic estimates. 
Theorem 1. For $n$ even,

$$
\begin{aligned}
& g_{n}^{(0)}=\left(1+O\left(n^{-1}\right)\right) g n^{-7 / 2} \rho^{-n} n !, \\
& g_{n}^{(1)}=\left(1+O\left(n^{-1}\right)\right) g_{c} n^{-7 / 2} \rho^{-n} n !,
\end{aligned}
$$

where all constants are analytically given, $\rho$ is the dominant singularity of $G^{(1)}(x)$, and $g_{c} / g=e^{-G^{(1)}(\rho)}$. Furthermore, $g_{n}^{(0)}=g_{n}^{(1)}=0$ for odd $n$.

We note that the first digits of $\gamma:=\rho^{-1}$ are 3.38, while the growth constant for the labeled simple cubic graphs is close to 3.13 4. The difference in growth is due to the fact that, unlike in the non-planar case, on average the cubic planar graphs contain a large number of multiple edges and loops.

Let $G_{n} \in \mathcal{G}_{n}^{(0)}$ denote a random graph chosen uniformly at random from the family $\mathcal{G}_{n}^{(0)}$ of all labeled cubic planar weighted multigraphs on $n$ vertices, where each multigraph with $f_{1}$ loops, $f_{2}$ double edges, and $f_{3}$ triple edges gets weight $2^{-f_{1}-f_{2}} 6^{-f_{3}}$. Using the asymptotic estimation of $g_{n}^{(0)}, g_{n}^{(1)}$, we obtain the following results on the size $L_{1}\left(G_{n}\right)$ of the largest component of $G_{n}$.

Lemma 2. (a) Uniformly over $n=1,2, \ldots$ and $0 \leq j<n / 2$,

$$
\mathbb{P}\left(L_{1}\left(G_{n}\right)=n-j\right)=(1+O(1 / n)) g_{c}(1-j / n)^{-7 / 2} j^{-7 / 2} .
$$

(b) There are constants $C$ and $n_{0}$ such that for all integers $n \geq n_{0}$ and $j=$ $1,2, \ldots$,

$$
\mathbb{P}\left(L_{1}\left(G_{n}\right) \leq n-j\right) \leq C j^{-5 / 2} .
$$

Proof. (a) If $L_{1}\left(G_{n}\right)=n-j$, where $j<n / 2$, then the graph obtained from $G_{n}$ by deleting the largest component (which is a connected cubic planar weighted multigraph on $n-j$ vertices) is an arbitrary cubic planar weighted multigraph on $j$ vertices. By Theorem 1 we have

$$
\begin{aligned}
\mathbb{P} & \left(L_{1}\left(G_{n}\right)=n-j\right)=\left(\begin{array}{c}
n \\
j
\end{array}\right) \frac{g_{n-j}^{(1)} \times g_{j}^{(0)}}{g_{n}^{(0)}} \\
& =(1+O(1 / n))\left(\begin{array}{c}
n \\
j
\end{array}\right) \frac{g_{c}(n-j)^{-7 / 2} \rho^{-(n-j)}(n-j) ! \times g j^{-7 / 2} \rho^{-j} j !}{g n^{-7 / 2} \rho^{-n} n !} \\
& =(1+O(1 / n)) g_{c}(1-j / n)^{-7 / 2} j^{-7 / 2} .
\end{aligned}
$$

(b) We let $R\left(G_{n}\right)$ denote $n-L_{1}\left(G_{n}\right)$, the number of vertices outside of the largest component. Part (a) implies that there is a constant $C^{\prime}$ such that for each $n$ and each $1 \leq j<n / 2$,

$$
\mathbb{P}\left(R\left(G_{n}\right)=j\right) \leq C^{\prime} j^{-7 / 2},
$$

and hence

$$
\mathbb{P}\left(j \leq R\left(G_{n}\right)<n / 2\right) \leq C^{\prime} \sum_{j \leq i<n / 2} j^{-7 / 2} \leq 2 C^{\prime} j^{-5 / 2} .
$$

Now it suffices to show that $\mathbb{P}\left(L_{1}\left(G_{n}\right) \leq n / 2\right)=O\left(n^{-5 / 2}\right)$. Let $G \in \mathcal{G}_{n}^{(0)}$ with $L_{1}(G) \leq n / 2$. Then, the vertex set of $G$ can be partitioned into two sets, $V_{1}$ and $V_{2}$, such that $n / 3 \leq\left|V_{1}\right|,\left|V_{2}\right| \leq 2 n / 3$, and $\left|V_{1}\right|+\left|V_{2}\right|=n$, and there is 
no vertex between $V_{1}$ and $V_{2}$. Hence, since for all sufficiently large $n$ we have $2^{-1} g n^{-7 / 2} \rho^{-n} n ! \leq\left|g_{n}^{(0)}\right| \leq 2 g n^{-7 / 2} \rho^{-n} n$ !, for large $n$ we get

$$
\begin{aligned}
\left|\left\{G \in \mathcal{G}_{n}^{(0)}: L_{1}(G) \leq n / 2\right\}\right| & \leq \sum_{n / 3 \leq i \leq n / 2}\left(\begin{array}{c}
n \\
i
\end{array}\right) g_{i}^{(0)} g_{n-i}^{(0)} \\
& \leq 4 g^{2} \rho^{-n} n ! \sum_{n / 3 \leq i \leq n / 2} i^{-7 / 2}(n-i)^{-7 / 2} \\
& =O\left(n^{-5 / 2} g_{n}^{(0)}\right) .
\end{aligned}
$$

This completes the proof of part (b).

3.2. Shrinking. Let $\mathcal{Q}(n ; d)$ denote the family of labeled planar multigraphs $G$ on vertex set $[n]=\{1,2, \ldots, n\}$ with $(3 n+d) / 2$ edges which have minimum degree at least three. Therefore the deficiency $\operatorname{df}(G)$ of $G \in \mathcal{Q}(n ; d)$ equals $d$. Moreover, let

$$
\mathbf{q}(n ; d)=\sum_{G \in \mathcal{Q}(n ; d)} 2^{-f_{1}(G)} \prod_{i \geq 2}(i !)^{-f_{i}(G)},
$$

where $f_{1}(G)$ counts loops in $G$ and $f_{i}(G)$ stands for the number of edges with parity $i$ for each $i \geq 2$. Since each $G \in \mathcal{Q}(n ; 0)$ is cubic, the asymptotic behavior of $\mathbf{q}(n ; 0)$ is determined by (2).

The following lemma gives bounds for $\mathbf{q}(n ; d)$ for $d \geq 1$.

Lemma 3. Let $1 \leq d \leq n$ and $n>0$ be integers such that $3 n+d$ is even. Then we have

$$
\frac{\mathbf{q}(n+d ; 0)}{d ! 6^{2 d}} \leq \mathbf{q}(n ; d) \leq \frac{\mathbf{q}(n+d ; 0) 9^{d}}{d !},
$$

where $\mathbf{q}(n ; 0)$ is given by (2).

Proof. The idea of the proof is to generate multigraphs in $\mathcal{Q}(n ; d)$ from the graphs from $\mathcal{Q}(n+d ; 0)$ by contracting edges incident to vertices $\{n+1, n+2, \ldots, n+d\}$. More precisely, for each of the vertices $\{n+1, n+2, \ldots, n+d\}$ we choose one of the incident edges $e_{i}=\left\{i, w_{i}\right\}, i=n+1, \ldots, n+d$, and contract it, i.e. we replace the vertices $i, w_{i}$ by one vertex $x$ which is adjacent to all neighbors of $i$ and $w_{i}$. Finally, we relabel $x$ by the label $\min \left\{i, w_{i}\right\}$. Sometimes this procedure fails to give a multigraph in $\mathcal{Q}(n+d ; 0)$ (e.g. when an edge is nominated by both of its ends or when some of the edges $e_{i}$ form a cycle), nonetheless each multigraph from $\mathcal{Q}(n ; d)$ can clearly be obtained from some graph from $\mathcal{Q}(n ; d)$ in the above process.

Now let us show the upper bound for $\mathbf{q}(n ; d)$. Choose $G \in \mathcal{Q}(n+d ; 0)$ and select edges $e_{i}, i=n+1, \ldots, n+d$, in one of at most $3^{d}$ ways. Suppose that by contracting all edges $e_{i}, i=n+1, \ldots, n+d$, we get a multigraph $H \in \mathcal{Q}(n ; d)$. Note that the weight of the multigraph $H$ could only increase by at most $(3 / 2)^{d}$ in the case when all vertices $i=n+1, \ldots, n+d$ belong to different components of size two (then we replace a triple edge which contributes to the weight $1 / 6$ by two loops of total weight $\left.(1 / 2)^{2}\right)$. Finally, we claim that there are at least $d ! 2^{-d}$ graphs $G^{\prime} \in \mathcal{Q}(n+d ; 0)$ which differ from $G$ only by labelings of vertices $n+1, \ldots, n+d$, i.e. each $H \in \mathcal{Q}(n ; d)$ is counted in this procedure at least $d ! 2^{-d}$ times. Indeed, let us remove all labels $n+1, \ldots, n+d$ from vertices of $G$ getting a graph $\bar{G}$ in which $d$ 'dummy' vertices are not labeled. We try to relabel those vertices with $n+1, \ldots, n+d$. Take any vertex $w$ of $H$ of degree larger than three. Then, $w$ is 
adjacent in $\bar{G}$ to $i$ dummy vertices, where $1 \leq i \leq 3$. Thus, we can label neighbors of $w$ with $i$ labels from $n+1, \ldots, n+d$ in at least

$$
\left(\begin{array}{l}
d \\
i
\end{array}\right) \geq \frac{d \ldots(d-i+1)}{2^{i}}
$$

ways. Now take another vertex which has already been labeled and choose labels for its dummy neighbors, and so on, until all dummy vertices get their labels. Clearly, the number of ways of doing that is bounded from below by $d ! 2^{-d}$. Hence, using (21), we get

$$
\mathbf{q}(n ; d) \leq \frac{\mathbf{q}(n+d ; 0) 3^{d}(3 / 2)^{d}}{d ! 2^{-d}}=\frac{\mathbf{q}(n+d ; 0) 9^{d}}{d !} .
$$

In order to get a lower bound for $\mathbf{q}(n ; d)$ we count only multigraphs $H \in \mathcal{Q}(n ; d)$ with maximum degree four. Note that the number of vertices of degree four in $H$ is $d$. Each vertex $v$ of degree four in $H$ we split into two vertices: one of them we label with $v$, and the other we leave as a 'dummy' vertex which has not been labeled so far. We add an edge between the two vertices, which we mark as used. We can make such a split into at most six possible ways. Now, we can choose labels for dummy vertices into one of $d$ ! possible ways (note that each dummy vertex is uniquely identified by the other end of the used edge). Consequently, from each $H$ we get at most $d ! 6^{d}$ different graphs from $\mathcal{Q}(n ; d)$ with $d$ disjoint edges marked. Note however that by splitting a vertex we may increase the weight of the graph by at most six (if we split the quadruple edge into two double edges), thus the total weight of different multigraphs obtained from $H$ is bounded from above by $d ! 6^{2 d}$. Consequently,

$$
\mathbf{q}(n ; d) \geq \frac{\mathbf{q}(n+d ; 0)}{d ! 6^{2 d}} .
$$

\section{Planar GRaphs With POSITIVE EXCESS}

Recall that a graph is called complex if all its components have positive excess. In this section, we derive the asymptotic number of all labeled complex planar graphs with given size and excess (Theorem 2). In order to do that we first estimate the number of such graphs with given deficiency (Lemma 4).

4.1. Fixed deficiency. In this section we estimate the number $C_{d}(k, k+\ell)$ of all labeled complex planar graphs $G$ on $k$ vertices with $\operatorname{ex}(G)=\ell>0$ and $\operatorname{df}(G)=$ $d \geq 0$.

To this end, first observe that the core $\operatorname{core}(G)$ of a complex graph $G$ can be obtained from $G$ by pruning each vertex of degree one in $G$ recursively, i.e. to get core $(G)$ we have to delete the tree-like part of $G$ rooted at vertices of $\operatorname{core}(G)$. Then, in order to find the $\operatorname{kernel} \operatorname{ker}(G)$ of $G$, one needs to replace each path in $\operatorname{core}(G)$ whose internal vertices are all of degree two by a single edge. Note that both $\operatorname{core}(G)$ and $\operatorname{ker}(G)$ have the same excess as $G$, and $\operatorname{ker}(G)$ is a planar multigraph of minimum degree at least three. Therefore, if $\operatorname{ex}(G)=\ell$ and $\operatorname{df}(G)=d$, the number of vertices in $\operatorname{ker}(G)$, denoted by $v($ ker $)$, equals $2 \ell-d$, and the number of edges, denoted by $e($ ker $)$, equals $3 \ell-d$.

In order to find $\operatorname{pl}(n, M)$ we reverse the above procedure and first count all possible kernels of graphs, then study the number of cores which lead to these kernels, and finally add to them the rooted forest to obtain all possible graphs 
$G$. More precisely, one can construct all graphs $G$ on $k$ vertices with $k+\ell$ edges, whose kernel is a planar multigraph graph with minimum degree at least three and deficiency $d$, in the following way:

(i) Choose the vertex set $\left\{c_{1}, c_{2}, \ldots, c_{i}\right\}$ of the core (for some $i \leq k$ ), and then select the vertex set $\left\{k_{1}, k_{2}, \ldots, k_{v(\text { ker })}\right\}$ of the kernel from the vertex set of the core. It can be done in $\left(\begin{array}{c}k \\ i\end{array}\right)\left(\begin{array}{c}i \\ v(\text { ker })\end{array}\right)$ ways.

(ii) Select a kernel of order $v$ (ker) among all the possible candidates for kernels (i.e. cubic planar weighted multigraphs on the vertex set $[v($ ker $)]$ with $e$ (ker) edges), and then map $[v($ ker $)]$ to $\left\{k_{1}, k_{2}, \ldots, k_{v(\text { ker })}\right\}$ in their relative order, i.e. we map $j$ to $k_{j}$. There are $\mathbf{q}(v($ ker $) ; d)$ ways of doing that.

(iii) Order the edges of the kernel lexicographically, each edge with a direction from one end point with the smaller label to the other end point with the larger label. For multiple edges with the same ends take any order, and choose one of two possible directions for each loop (the weights we assign when we counted candidates for the kernel was chosen precisely to assure that in this way we avoid double counting). Now make a directed path of length $e$ (ker) consisting of the kernel edges, according to this order, and insert the core vertices that are not in the kernel on the edges of the kernel in such a way that each loop gets at least two core vertices, and at least $j-1$ edges from $j$ edges incident to the common end points to get at least one core vertex. Let $m=m(\ell)$ be such that

$$
m \ell=2 f_{1}+\sum_{j \geq 2}(j-1) f_{j},
$$

where $f_{1}$ denotes the number of loops and $f_{j}$ multiple edges with parity $j$ for $j \geq 2$ in $\operatorname{ker}(G)$ (hence $0 \leq m \leq 6)$. This can be done in $(i-$ $v($ ker $)) !\left(\begin{array}{c}i-v(\text { ker })-m \ell+e(\text { ker })-1 \\ e(\text { ker })-1\end{array}\right)$ ways.

(iv) Plant a rooted forest on the core vertices. According to Cayley's formula, one can do it in $i k^{k-i-1}$ ways.

As a consequence, we have

$$
\begin{aligned}
& C_{d}(k, k+\ell) \\
& =\sum_{i}\left(\begin{array}{l}
k \\
i
\end{array}\right)\left(\begin{array}{c}
i \\
v(\mathrm{ker})
\end{array}\right) \mathbf{q}(v(\mathrm{ker}) ; d)(i-v(\mathrm{ker})) !\left(\begin{array}{c}
i-v(\mathrm{ker})-m \ell+e(\mathrm{ker})-1 \\
e(\mathrm{ker})-1
\end{array}\right) i k^{k-i-1}
\end{aligned}
$$

$$
=\sum_{i} \frac{(k)_{i}}{(2 \ell-d) !} \mathbf{q}(2 \ell-d ; d)\left(\begin{array}{c}
i+\ell-m \ell-1 \\
3 \ell-d-1
\end{array}\right) i k^{k-i-1}
$$

Applying Lemma 3 we obtain the following estimate.

Lemma 4. Let $d \geq 0$ and $k, \ell>0$ be integers. Let $\gamma, g$ be the constants so that the assertion of Theorem 1 holds. Then

$$
\begin{aligned}
C_{d}(k, k+\ell) & =2^{-4} g k^{k+\frac{3 \ell-d-1}{2}} \gamma^{2 \ell} \ell^{-7 / 2} e^{\frac{3 \ell-d}{2}}(3 \ell-d)^{-\frac{3 \ell-d-1}{2}} \alpha^{d}\left(\begin{array}{c}
2 \ell \\
d
\end{array}\right) \\
& \times \exp \left(\left(\frac{2}{3} d-(m+1) \ell\right) \sqrt{\frac{3 \ell-d}{k}}+O\left(\frac{\ell^{2}}{k}+\frac{1}{\ell}\right)\right),
\end{aligned}
$$

for some $\alpha=\alpha(k, \ell), 6^{-2} \leq \alpha \leq 9$, and $m=m(k, \ell), 0 \leq m \leq 6$. 
Moreover, the typical size of the core in a randomly chosen complex planar graph $G$ on $k$ vertices with $\operatorname{ex}(G)=\ell$ and $\operatorname{df}(G)=d$ is a.a.s.

$$
(1+O(\sqrt{\ell / k})+O(1 / \sqrt{\ell})) \sqrt{3 k \ell} .
$$

Proof. Note that from Lemma 3 we have

$$
\frac{\mathbf{q}(2 \ell ; 0)}{d ! 6^{2 d}} \leq \mathbf{q}(2 \ell-d ; d) \leq \frac{\mathbf{q}(2 \ell ; 0) 9^{d}}{d !},
$$

where

$$
\mathbf{q}(2 \ell ; 0) \stackrel{\text { 尚 }}{=}\left(1+o\left(\ell^{-1}\right)\right) g(2 \ell)^{-7 / 2} \gamma^{2 \ell}(2 \ell) !
$$

Therefore from (7) we get

(8)

$$
\begin{aligned}
& \sum_{2 \ell-d \leq i \leq k} g k^{k-1} \gamma^{2 \ell}(2 \ell)^{-7 / 2} 6^{-2 d}\left(\begin{array}{c}
2 \ell \\
d
\end{array}\right) \sum_{i}(k)_{i} i k^{-i}\left(\begin{array}{c}
i+(1-m) \ell-1 \\
3 \ell-d-1
\end{array}\right) \\
& \leq C_{d}(k, k+\ell) \\
& \leq \sum_{2 \ell-d \leq i \leq k} g k^{k-1} \gamma^{2 \ell}(2 \ell)^{-7 / 2} 9^{d}\left(\begin{array}{c}
2 \ell \\
d
\end{array}\right) \sum_{i}(k)_{i} i k^{-i}\left(\begin{array}{c}
i+(1-m) \ell-1 \\
3 \ell-d-1
\end{array}\right) .
\end{aligned}
$$

Thus, it is enough to estimate the quantity

(9) $\tilde{C}_{d}(k, k+\ell)=g k^{k-1} \gamma^{2 \ell}(2 \ell)^{-7 / 2} \alpha^{d}\left(\begin{array}{c}2 \ell \\ d\end{array}\right) \sum_{i}(k)_{i} i k^{-i}\left(\begin{array}{c}i+(1-m) \ell-1 \\ 3 \ell-d-1\end{array}\right)$,

where $\alpha$ and $m$ satisfy $6^{-2} \leq \alpha \leq 9$ and $0 \leq m \leq 6$.

Below we use Stirling's formula several times,

$$
n !=(1+O(1 / n)) \sqrt{2 \pi} n^{n+1 / 2} e^{-n} \quad \text { for } \quad n \in \mathbb{N},
$$

and the following consequence of the Maclaurin expansion of $e^{x}$ :

$$
1+x=\exp \left(x-x^{2} / 2+x^{3} / 3+O\left(x^{4}\right)\right) .
$$

To derive an asymptotic formula for (9), note that

$$
\begin{aligned}
(k)_{i}=k^{i} \prod_{j=0}^{i-1}\left(1-\frac{j}{k}\right) & \stackrel{\prod 1}{=} k^{i} \exp \left(\sum_{j=0}^{i-1}\left(-\frac{j}{k}-\frac{1}{2}\left(\frac{j}{k}\right)^{2}-\frac{1}{3}\left(\frac{j}{k}\right)^{3}+O\left(\frac{j}{k}\right)^{4}\right)\right) \\
& =k^{i} \exp \left(-\frac{i^{2}}{2 k}-\frac{i^{3}}{6 k^{2}}+O\left(\frac{i}{k}+\frac{i^{4}}{k^{3}}\right)\right)
\end{aligned}
$$

and

$$
\begin{aligned}
& \left(\begin{array}{c}
i+(1-m) \ell-1 \\
3 \ell-d-1
\end{array}\right)=\frac{(i+(1-m) \ell-1)_{3 \ell-d-1}}{(3 \ell-d-1) !} \\
& \stackrel{(100)}{=} \frac{(3 \ell-d) i^{3 \ell-d-1} \prod_{j=1}^{3 \ell-d-1}\left(1+\frac{(1-m) \ell-j}{i}\right)}{(1+O(1 / \ell)) \sqrt{2 \pi(3 \ell-d)}((3 \ell-d) / e)^{3 \ell-d}} \\
& \stackrel{(11)}{=} \frac{e^{3 \ell-d} i^{3 \ell-d-1}}{\sqrt{2 \pi}(3 \ell-d)^{3 \ell-d-1 / 2}} \exp \left(-\frac{(3 \ell-d)^{2}}{2 i}+\frac{(1-m) \ell(3 \ell-d)}{i}+\frac{1}{\ell}+\frac{\ell}{i}\right) .
\end{aligned}
$$


Next, we rewrite the sum over $i$ in (9) as

$$
\sum_{i}(k)_{i} i k^{-i}\left(\begin{array}{c}
i+(1-m) \ell-1 \\
3 \ell-d-1
\end{array}\right)=\frac{e^{3 \ell-d}}{\sqrt{2 \pi}(3 \ell-d)^{3 \ell-d-1 / 2}} \sum_{i} \exp (a(i)),
$$

where the function $a(i)=a_{k, \ell, d}(i)$ is defined as

$$
\begin{aligned}
a(i)=(3 \ell-d) \log i & -\frac{i^{2}}{2 k}-\frac{i^{3}}{6 k^{2}}-\frac{(3 \ell-d)^{2}}{2 i}+\frac{(1-m) \ell(3 \ell-d)}{i}+\frac{1}{\ell} \\
& +\frac{\ell}{i}+O\left(\frac{i}{k}\right)+O\left(\frac{i^{4}}{k^{3}}\right) .
\end{aligned}
$$

We observe that the main contribution to (12) comes from the terms $i=i_{0}+O(\sqrt{k})$, where

$$
i_{0}=\left(1+\frac{m-1}{6} \sqrt{\frac{3 \ell-d}{k}}\right) \sqrt{k(3 \ell-d)} .
$$

For such $i$ 's, we have

$$
\begin{aligned}
\exp \left(a\left(i_{0}\right)\right)=(k(3 \ell-d))^{\frac{3 \ell-d}{2}} \exp & \left(-\frac{3 \ell-d}{2}+\left(\frac{2}{3} d-(m+1) \ell\right) \sqrt{\frac{3 \ell-d}{k}}\right) \\
& \times \exp \left(O\left(\frac{\ell^{2}}{k}\right)+O\left(\frac{1}{\ell}\right)\right)
\end{aligned}
$$

and

$$
\sum_{i=i_{0}+O(\sqrt{k})} \exp \left(a(i)-a\left(i_{0}\right)\right)=\sum_{\Delta i=O(\sqrt{k})} \exp \left(-\frac{1}{k}(\Delta i)^{2}+O\left(\frac{\ell}{k}\right)\right)=\sqrt{\pi k} \exp \left(O\left(\frac{\ell}{k}\right)\right) .
$$

Thus, we get

$$
\begin{aligned}
& \sum_{i}(k)_{i} i k^{-i}\left(\begin{array}{c}
i+(1-m) \ell-1 \\
3 \ell-d-1
\end{array}\right)=2^{-1 / 2} k^{\frac{3 \ell-d+1}{2}}(3 \ell-d)^{-\frac{3 \ell-d+1}{2}} e^{\frac{3 \ell-d}{2}} \\
& \times \exp \left(\left(\frac{2}{3} d-(m+1) \ell\right) \sqrt{\frac{3 \ell-d}{k}}+O\left(\frac{\ell^{2}}{k}+\frac{1}{\ell}\right)\right) .
\end{aligned}
$$

Finally, (9) and (14) yield

$$
\begin{aligned}
\tilde{C}_{d}(k, k+\ell)=2^{-4} g \gamma^{2 \ell} & \ell^{-7 / 2} k^{k+\frac{3 \ell-d-1}{2}} e^{\frac{3 \ell-d}{2}}(3 \ell-d)^{-\frac{3 \ell-d-1}{2}} \alpha^{d}\left(\begin{array}{c}
2 \ell \\
d
\end{array}\right) \\
& \times \exp \left(\left(\frac{2}{3} d-(m+1) \ell\right) \sqrt{\frac{3 \ell-d}{k}}+O\left(\frac{\ell^{2}}{k}+\frac{1}{\ell}\right)\right) .
\end{aligned}
$$

The last part of the assertion follows from the fact that the main contribution to the sum (9) comes from the terms $i=(1+o(1)) i_{0}$, where $i_{0}$ is given by (13).

4.2. Asymptotic numbers and typical deficiency. In this section we estimate the number $C(k, k+\ell)$ of labeled complex planar graphs with $k$ vertices and $k+\ell$ edges.

Theorem 2. Let $\gamma, g, g_{c}$ be the constants for which the assertion of Theorem 1 holds and let $k, \ell>0$ be integers. 
(i) There exists a function $\beta=\beta(k, \ell)$ with $-14 \leq \beta \leq 2^{7}$, for which

$$
\begin{aligned}
C(k, k+\ell)=2^{-4} 3^{1 / 2} g & k^{k+3 \ell / 2-1 / 2}\left(\frac{\gamma^{2} e^{3 / 2}}{3^{3 / 2}}\right)^{\ell} \ell^{-3 \ell / 2-3} \\
& \times \exp \left(\beta \sqrt{\frac{\ell^{3}}{k}}+O\left(\frac{\ell^{2}}{k}\right)+O\left(\frac{1}{\ell}\right)\right) .
\end{aligned}
$$

(ii) The number $C^{\text {conn }}(k, k+\ell)$ of labeled connected complex planar graphs with $k$ vertices and $k+\ell$ edges is given by a similar formula, with $g$ replaced by $g_{c}$.

(iii) A graph chosen uniformly at random among all complex planar graphs with $k$ vertices and $k+\ell$ edges has a.a.s. deficiency $\Theta\left(\sqrt{\ell^{3} / k}\right)$ and the core of size $(1+O(\sqrt{\ell / k})+O(1 / \sqrt{\ell})) \sqrt{3 k \ell}$. In particular, if $\ell=o\left(k^{1 / 3}\right)$, then a.a.s. such a random graph is clean.

(iv) If $\ell=O\left(k^{1 / 3}\right)$, then a graph chosen uniformly at random among all complex planar graphs with $k$ vertices and $k+\ell$ edges has a.a.s. $O(1)$ components, among which there is a giant component of size $k-O(k / \ell)$. Furthermore, a.a.s. each small component has $\Theta(k / \ell)$ vertices and the probability that such a graph contains exactly $h$ such components is bounded away from both 0 and 1 for every $h=0,1, \ldots$.

Proof. Using the asymptotic estimate of $C_{d}(k, k+\ell)$ from Lemma 4, we get the following:

$$
\begin{aligned}
C(k, k+\ell) & =\sum_{d} C_{d}(k, k+\ell) \\
& \stackrel{8}{=} 2^{-4} g \gamma^{2 \ell} k^{k+\frac{3 \ell-1}{2}} e^{\frac{3 \ell}{2}}(3 \ell)^{-\frac{3 \ell-1}{2}} \ell^{-7 / 2} \exp \left(O\left(\frac{\ell^{2}}{k}+\frac{1}{\ell}\right)\right) \\
& \times \sum_{d} k^{-\frac{d}{2}}(3 \ell)^{\frac{d}{2}} \alpha^{d}\left(\begin{array}{c}
2 \ell \\
d
\end{array}\right) \exp \left(\left(\frac{2}{3} d-(m+1) \ell\right) \sqrt{\frac{3 \ell-d}{k}}\right),
\end{aligned}
$$

where $\alpha=\alpha(k, \ell)$ and $m=m(k, \ell)$ satisfy $6^{-2} \leq \alpha \leq 9$ and $0 \leq m \leq 6$.

Define a function $\eta(d)=\eta_{k, \ell}(d)$ as

$$
\eta(d)=\sum_{d} k^{-\frac{d}{2}}(3 \ell)^{\frac{d}{2}} \alpha^{d}\left(\begin{array}{c}
2 \ell \\
d
\end{array}\right) \exp \left(\left(\frac{2}{3} d-(m+1) \ell\right) \sqrt{\frac{3 \ell-d}{k}}\right) .
$$

We observe that the main contribution to $\eta(d)$ comes from $d=\Theta\left(\sqrt{\ell^{3} / k}\right)$, and therefore

$$
\begin{aligned}
\eta(d) & =\sum_{d}\left(\begin{array}{c}
2 \ell \\
d
\end{array}\right)(\alpha \sqrt{3 \ell / k})^{d} \exp \left(-(m+1) \ell \sqrt{\frac{3 \ell}{k}}+O\left(\frac{\ell^{2}}{k}\right)\right) \\
& =(1+\alpha \sqrt{3 \ell / k})^{2 \ell} \exp \left(-(m+1) \sqrt{\frac{3 \ell^{3}}{k}}+O\left(\frac{\ell^{2}}{k}\right)\right) \\
& =\exp \left((2 \alpha-(m+1)) \sqrt{\frac{3 \ell^{3}}{k}}+O\left(\frac{\ell^{2}}{k}\right)\right) .
\end{aligned}
$$

Finally, taking $\beta=\beta(k, \ell)=(2 \alpha-(m+1)) \sqrt{3}$ (and thus $-14 \leq \beta \leq 2^{7}$ ) completes the proof of (i). 
In order to show (ii) one should repeat computations from the proof of Lemma 4 and the one given above, for graphs with connected kernels. Therefore $\mathbf{q}(2 \ell ; 0)$ should be replaced by the number of connected cubic planar weighted multigraphs vertices, which, by (3), is equal to $\left(1+O\left(\ell^{-1}\right)\right) g_{c}(2 \ell)^{-7 / 2} \gamma^{2 \ell}(2 \ell)$ !.

To see (iii) observe that the main contribution to (15) comes from $d=\Theta\left(\sqrt{\ell^{3} / k}\right)$.

Finally, Lemma 2 states that a randomly chosen cubic planar graph a.a.s. contains a giant component of size $n-O(1)$, and using exact counts it is easy to show that the number of small components has a non-degenerate distribution. Since for $\ell=O\left(k^{1 / 3}\right)$ we can count graphs up to a constant factor, a similar statement is also true for supercubic weighted multigraphs. Now (iv) follows from the fact that the trees rooted in one edge of the kernel have on average $\Theta(k / \ell)$ vertices altogether.

Unfortunately, since we can only estimate the number of supercubic graphs up to a factor of $\exp \left(\sqrt{\ell^{3} / k}\right)$, we cannot prove the assertion of Theorem 2(iv) in the case when $\ell \gg k^{1 / 3}$. Nonetheless, we think that it is also true in a much wider range and that the following conjecture holds.

Giant Conjecture. The assertion of Theorem 2(iv) holds for every $\ell \leq k$.

\section{EVOLUTION OF PLANAR GRAPHS}

In this section we derive the asymptotic number $\mathrm{pl}(n, M)$ of labeled planar graphs with $n$ vertices and $M$ edges and investigate how the size of the largest component in $P(n, M)$, its excess, and the size of its core change with $M$.

Throughout the section, we let $\gamma, g$ be the constants for which the assertion of Theorem 1 holds. By $L_{j}(n, M)$ we denote the number of vertices in the $j$-th largest component of $P(n, M)$. Let $\operatorname{ex}_{\mathrm{c}}(n, M)$ (resp. $\left.\mathrm{cr}_{\mathrm{c}}(n, M)\right)$ stand for the excess (resp. the number of vertices in the core) of the subgraph of $P(n, M)$ which consists of its complex components, and let $L_{\mathrm{c}}(n, M)$ denote its size. Finally, let $\operatorname{ex}(n, M)$ and $\operatorname{cr}(n, M)$ denote the excess and the size of the core of the largest component of $P(n, M)$, respectively.

Before studying $P(n, M)$ we recall in the next section some properties of the uniform random graph $G(n, M)$ which are relevant for our argument.

5.1. Properties of the uniform random graph. Let $\bar{L}_{j}(n, M)$ denote the number of vertices in the $j$-th largest component of $G(n, M)$, let $\operatorname{ex}(n, M)$ stand for the excess of the largest component of $G(n, M)$, and let $\overline{c r}(n, M)$ be the number of vertices in the core of the largest component of $G(n, M)$.

The following results on the largest components were proved by Luczak 21, 23. and Łuczak, Pittel, and Wierman [25] (see also Janson, Łuczak, and Ruciński [20]).

Theorem 3 (Subcritical phase). Let $M=n / 2+s$, where $s=s(n)$. If $s^{3} / n^{2} \rightarrow$ $-\infty$, then for $j$ fixed, a.a.s. $\bar{L}_{j}(n, M)=(1 / 2+o(1)) \frac{n^{2}}{s^{2}} \log \frac{|s|^{3}}{n^{2}}$. Furthermore, a.a.s. the $j$-th largest component of $G(n, M)$ is a tree.

Theorem 4 (Critical phase). Let $M=n / 2+s$, where $s=s(n)$. If $s^{3} / n^{2} \rightarrow c$, then for $j$ fixed, a.a.s. $\bar{L}_{j}(n, M)=\Theta\left(n^{2 / 3}\right)$. The total excess of the complex components of $G(n, M)$ is a.a.s. $O(1)$, and the probability that the $j$-th largest component of $G(n, M)$ has excess $h$ is bounded away from zero for every fixed $j=1,2, \ldots$ and $h=-1,0,1, \ldots$ 
Furthermore, if $G(n, M)$ contains some complex components, then a.a.s. they have $\Theta\left(n^{2 / 3}\right)$ vertices in total and $\overline{\operatorname{cr}}(n, M)=\Theta\left(n^{1 / 3}\right)$.

Theorem 5 (Supercritical phase). Let $M=n / 2+s$, where $s=s(n)$. If $s^{3} / n^{2} \rightarrow$ $\infty$, then a.a.s. $\bar{L}_{1}(n, M)=(4+o(1)) s$, while for $j \geq 2$ fixed, $\bar{L}_{j}(n, M)=(1 / 2+$ $o(1)) \frac{n^{2}}{s^{2}} \log \frac{s^{3}}{n^{2}}$. Moreover, a.a.s. the $j$-th largest component of $G(n, M)$ is a tree, provided $j \geq 2$.

The structure of the giant component of $G(n, M)$ was studied by Luczak [22].

Theorem 6. If $M=n / 2+s$, where $s^{3} / n^{2} \rightarrow \infty$ but $s=o(n)$, then a.a.s. $\overline{\mathrm{ex}}(n, M)=(16 / 3+o(1)) \frac{s^{3}}{n^{2}}$ and $\overline{\mathrm{cr}}(n, M)=(8+o(1)) \frac{s^{2}}{n}$.

The following threshold for the property that $G(n, M)$ is planar was proved by Łuczak, Pittel, and Wierman [25].

Theorem 7 (Planarity). Let $M=n / 2+c n^{2 / 3}$ for a constant $c$. Then the probability that $G(n, M)$ is planar tends to a limit $\varphi(c)$ as $n \rightarrow \infty$, where $0<\varphi(c)<1$, $\lim _{c \rightarrow-\infty} \varphi(c)=1$, and $\lim _{c \rightarrow \infty} \varphi(c)=0$.

A different proof of the above result can be found in Janson et al. [19, where it is also shown that $0.987<\varphi(0)<0.9998$.

We shall use Theorems 3, 4 and 7 in the proofs of Theorems 9 and 10 , We do not use Theorems 5 and 6 in our proofs below, but we decided to invoke them here to show how our results differ from those for $G(n, M)$.

For a constant $c \in(-\infty, \infty)$, let us define

$$
\nu(c)=\sqrt{\frac{2}{3 \pi}} e^{-4 c^{3} / 3} \sum_{r=0}^{\infty} \frac{\left(-9 c^{3}\right)^{r / 3}}{r !} \Gamma\left(\frac{2 r}{3}+\frac{1}{2}\right) \cos \frac{\pi r}{3} .
$$

Note that $\nu(c)$ decreases monotonically with $\nu(c) \rightarrow 1$ as $c \rightarrow-\infty$ and $\nu(c) \leq$ $\exp \left(-(4+o(1)) c^{3} / 3\right)$ for large $c$. In our argument we also use the following result of Britikov [7]. Here and below by $\rho(n, M)$ we denote the probability that $G(n, M)$ contains no complex component. In other words, $\rho(n, M)=U(n, M) /\left(\begin{array}{c}n \\ 2 \\ M\end{array}\right)$, where $U(n, M)$ denotes the number of labeled graphs with $n$ vertices and $M$ edges, which contain no complex components.

Theorem 8. Let $M=n / 2+s$, where $s=s(n)$. Then the following holds:

(i) If $s^{3} / n^{2} \rightarrow-\infty$, then $\rho(n, M)=1+O\left(n^{2} /|s|^{3}\right)$.

(ii) If $s^{3} / n^{2} \rightarrow c$, where $c$ is a (not necessarily positive) constant, then $\rho(n, M)$ $=(1+o(1)) \nu\left(c^{1 / 3}\right)$.

(iii) If $s^{3} / n^{2} \rightarrow \infty$, then $\rho(n, M) \leq \exp \left(-s^{3} / n^{2}\right)$.

5.2. The formula for $\operatorname{pl}(n, M)$. The main ingredient of our argument is a simple observation that each graph can be uniquely decomposed into the complex part and the remaining part which consists of isolated trees and unicyclic components. Moreover, it is the complex part which determines whether the graph is planar. Consequently, the number $\operatorname{pl}(n, M)$ of labeled planar graphs on $n$ vertices with $M$ edges is given by

$$
\operatorname{pl}(n, M)=\sum_{k, \ell}\left(\begin{array}{l}
n \\
k
\end{array}\right) C(k, k+\ell) U(n-k, M-k-\ell) .
$$


Thus, the estimate of $C(k, k+\ell)$ (Theorem 2) and that of $U(n-k, M-k-\ell$ ) (Theorem 8) yield the asymptotic estimate of $\mathrm{pl}(n, M)$. Moreover, the leading terms of (17) give us information on the size of the complex part of the graph and thus, by Theorem 2, on the size of the largest component of $P(n, M)$ and its internal structure. On the other hand, the size of the largest non-complex component can be deduced from Theorems 3 and 4

5.3. Subcritical phase. The behavior of $P(n, M)$ in the subcritical case follows directly from Theorem 3 .

Theorem 9. Let $M=n / 2+s$, where $s=o(n)$. If $s^{3} / n^{2} \rightarrow-\infty$, then

$$
\operatorname{pl}(n, M)=(1+o(1)) \frac{n^{n+2 s} e^{n / 2+s-1 / 2}}{\sqrt{\pi}(n+2 s)^{n / 2+s+1 / 2}} .
$$

Furthermore, for fixed $j$ a.a.s. $L_{j}(n, M)=(1 / 2+o(1)) \frac{n^{2}}{s^{2}} \log \frac{|s|^{3}}{n^{2}}$ and the $j$-th largest component of $P(n, M)$ is a tree.

Proof. Theorem 7 states that a.a.s. $G(n, M)$ is planar. Therefore, we have

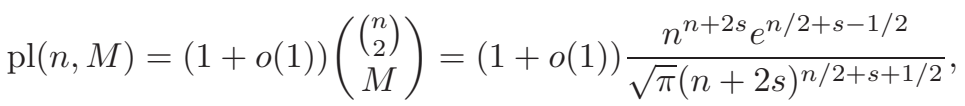

and the structure of $P(n, M)$ follows from Theorem 3 .

5.4. Critical phase. The critical period is only slightly harder to deal with than the previous one as far as we estimate $\operatorname{pl}(n, M)$ only up to a constant factor.

Theorem 10. Let $M=n / 2+s$. If $s^{3} / n^{2} \rightarrow c$ for a constant $c \in(-\infty, \infty)$, then

$$
\operatorname{pl}(n, M)=\Theta(1) \frac{n^{n+2 s} e^{n / 2+s-1 / 2}}{\sqrt{\pi}(n+2 s)^{n / 2+s+1 / 2}} .
$$

Furthermore, for $j$ fixed, a.a.s. $L_{j}(n, M)=\Theta\left(n^{2 / 3}\right)$, and if $P(n, M)$ contains complex components, then $L_{\mathrm{c}}(n, M)=\Theta\left(n^{2 / 3}\right), \operatorname{ex}(n, M)=O(1)$, and $\operatorname{cr}(n, M)=$ $\Theta\left(n^{1 / 3}\right)$.

Proof. Theorem 7 states that the probability that $G(n, M)$ is planar tends to a limit which is strictly between 0 and 1 . Hence

$$
\operatorname{pl}(n, M)=\Theta(1)\left(\begin{array}{c}
n \\
2 \\
M
\end{array}\right)=\Theta(1) \frac{n^{n+2 s} e^{n / 2+s-1 / 2}}{(n+2 s)^{n / 2+s+1 / 2}} .
$$

The assertion on the structure of $P(n, M)$ is a direct consequence of Theorem 4 .

5.5. Supercritical phase. The evolution of $P(n, M)$ in the 'early supercritical' period starts to be more interesting. Note that the result below estimates $\operatorname{pl}(n, M)$ up to a factor of $1+o(1)$.

Theorem 11. Let $M=n / 2+s$, where $s=o(n)$ and $s^{3} / n^{2} \rightarrow \infty$. Then

$$
\begin{gathered}
\operatorname{pl}(n, M)=(1+o(1)) \frac{g 3^{5 / 2}}{2^{7} \sqrt{\pi} \gamma^{10 / 3} e^{3 / 4}} \frac{n^{n+11 / 6}}{s^{7 / 2}} \frac{e^{n / 2-s}}{(n-2 s)^{n / 2-s}} \exp \left(\frac{\gamma^{4 / 3} s}{n^{2 / 3}}\right) \\
\times \int_{-\infty}^{\infty} \exp \left(-\frac{x^{3}}{6}+\frac{\gamma^{4 / 3} x}{2}\right) \nu\left(-\frac{x}{2}\right) d x .
\end{gathered}
$$


Furthermore, a.a.s. $L_{1}(n, M)=(2+o(1)) s$, while for any fixed $j \geq 2$ we have $L_{j}(n, M)=\Theta\left(n^{2 / 3}\right)$. In addition, a.a.s. $\operatorname{ex}(n, M)=\left(\frac{2 \gamma^{4 / 3}}{3}+o(1)\right) \frac{s}{n^{2 / 3}}$ and $\operatorname{cr}(n, M)$ $=\left(2 \gamma^{2 / 3}+o(1)\right) \frac{s}{n^{1 / 3}}$.

Proof. From Theorem 2 and (10), we get

$$
\begin{aligned}
\left(\begin{array}{l}
n \\
k
\end{array}\right) & \stackrel{(10)}{=} \frac{(1+O(1 / k))}{\sqrt{2 \pi}} \frac{n^{n+1 / 2}}{(n-k)^{n-k+1 / 2} k^{k+1 / 2}}, \\
C(k, k+\ell) & =\frac{g 3^{1 / 2}}{2^{4}} k^{k+3 \ell / 2-1 / 2}\left(\frac{\gamma^{2} e^{3 / 2}}{3^{3 / 2}}\right)^{\ell} \ell^{-3 \ell / 2-3} \\
& \times \exp \left(\beta \sqrt{\frac{\ell^{3}}{k}}+O\left(\frac{\ell^{2}}{k}\right)+O\left(\frac{1}{\ell}\right)\right) .
\end{aligned}
$$

In addition, the estimate

$$
\left(\begin{array}{c}
\left(\begin{array}{c}
n \\
2
\end{array}\right) \\
j
\end{array}\right)=\frac{n^{2 j}}{\sqrt{\pi}(2 j)^{j+1 / 2}} \exp \left(j-\frac{j}{n}-\frac{j^{2}}{n^{2}}+O\left(\frac{1}{n}\right)+O\left(\frac{j}{n^{2}}\right)\right)
$$

gives

$$
\begin{aligned}
& U(n-k, M-k-\ell) \\
&= \rho(n-k, M-k-\ell)\left(\begin{array}{c}
\left(\begin{array}{c}
n-k \\
2
\end{array}\right) \\
M-k-\ell
\end{array}\right) \\
&=(1+O(1 / n)) \frac{\rho(n-k, n / 2+s-k-\ell)}{\sqrt{\pi} e^{3 / 4}} \frac{e^{n / 2+s-k}(n-k)^{n+2 s-2 k}}{(n+2 s-2 k)^{n / 2+s-k+1 / 2}} \\
& \times\left(\frac{n+2 s-2 k}{(n-k)^{2}}\right)^{\ell} .
\end{aligned}
$$

Therefore, we get

$$
\begin{aligned}
\operatorname{pl}(n, M)= & \sum_{k, \ell}\left(\begin{array}{l}
n \\
k
\end{array}\right) C(k, k+\ell) U(n-k, M-k-\ell) \\
= & (1+O(1 / n)) \frac{g 3^{1 / 2}}{2^{9 / 2} \pi e^{3 / 4}} n^{n-1 / 2} e^{n / 2+s} \\
& \times \sum_{k}(1+O(1 / k)+O(k / n)) \frac{\rho(n-k, n / 2+s-k)(n-k)^{2 s-k}}{k e^{k}(n+2 s-2 k)^{n / 2+s-k}} \\
& \times \sum_{\ell}\left(\frac{\gamma^{2} e^{3 / 2} k^{3 / 2}(n+2 s-2 k)}{3^{3 / 2}(n-k)^{2} \ell^{3 / 2}}\right)^{\ell} \ell^{-3} \\
& \times \exp \left(\beta \sqrt{\frac{\ell^{3}}{k}}+O\left(\frac{\ell^{2}}{k}\right)+O\left(\frac{1}{\ell}\right)\right) .
\end{aligned}
$$

Now let $\phi=\phi(n, s, k):=\frac{\gamma^{2} e^{3 / 2} k^{3 / 2}(n+2 s-2 k)}{3^{3 / 2}(n-k)^{2}}$. Then the sum in (18) depending on $\ell$ becomes

$$
\sum_{\ell \geq 1}\left(\frac{\phi}{\ell^{3 / 2}}\right)^{\ell} \ell^{-3}=\sum_{\ell \geq 1} \ell^{-3} \exp (b(\ell))
$$


where the function $b(\ell)=b_{n, s, k}(\ell)$ is defined as

$$
b(\ell)=\ell \log \phi-\frac{3}{2} \ell \log \ell .
$$

The main contribution to (19) comes from the terms $\ell=\ell_{0}+O\left(\sqrt{\ell_{0}}\right)$, where

$$
\ell_{0}=\ell_{0}(n, s, k):=e^{-1} \phi^{2 / 3}=\frac{\gamma^{4 / 3}}{3} \frac{k(n+2 s-2 k)^{2 / 3}}{(n-k)^{4 / 3}} .
$$

Furthermore, we have

$$
\begin{aligned}
\ell_{0}^{-3} \exp \left(b\left(\ell_{0}\right)\right) & =\ell_{0}^{-3} \exp \left(3 \ell_{0}\left(\log \phi^{2 / 3}-\log \ell_{0}\right) / 2\right) \\
& =\frac{3^{3}}{\gamma^{4}} \frac{(n-k)^{4}}{k^{3}(n+2 s-2 k)^{2}} \exp \left(\frac{\gamma^{4 / 3}}{2} \frac{k(n+2 s-2 k)^{2 / 3}}{(n-k)^{4 / 3}}\right)
\end{aligned}
$$

and

$$
\begin{aligned}
\sum_{\ell=\ell_{0}+O\left(\sqrt{\ell_{0}}\right)} \exp \left(b(\ell)-b\left(\ell_{0}\right)\right) & =(1+o(1)) \sum_{\ell=\ell_{0}+O\left(\sqrt{\ell_{0}}\right)} \exp \left(-\frac{3\left(\ell-\ell_{0}\right)^{2}}{4 \ell_{0}}\right) \\
& =(1+o(1)) \sqrt{\frac{4 \pi \ell_{0}}{3}} \\
& =(1+o(1)) \frac{2 \pi^{1 / 2} \gamma^{2 / 3}}{3} \frac{k^{1 / 2}(n+2 s-2 k)^{1 / 3}}{(n-k)^{2 / 3}} .
\end{aligned}
$$

This implies

$$
\begin{aligned}
\sum_{\ell \geq 1}\left(\frac{\phi}{\ell^{3 / 2}}\right)^{\ell} \ell^{-3}= & (1+o(1)) \ell_{0}^{-3} \exp \left(b\left(\ell_{0}\right)\right) \sum_{\ell=\ell_{0}+O\left(\sqrt{\ell_{0}}\right)} \exp \left(b(\ell)-b\left(\ell_{0}\right)\right) \\
=(1+ & o(1)) \frac{2 \sqrt{\pi} 3^{2}}{\gamma^{10 / 3}} \frac{(n-k)^{10 / 3}}{k^{5 / 2}(n+2 s-2 k)^{5 / 3}} \\
& \times \exp \left(\frac{\gamma^{4 / 3} k}{2}\left(\frac{(n+2 s-2 k)}{(n-k)^{2}}\right)^{2 / 3}\right)
\end{aligned}
$$

and hence (18) becomes

$$
\begin{aligned}
\operatorname{pl}(n, n / 2+s)= & (1+O(1 / n)) \frac{g 3^{5 / 2}}{2^{7 / 2} \pi^{1 / 2} e^{3 / 4} \gamma^{10 / 3}} n^{n+7 / 6} e^{n / 2+s} \\
& \times \sum_{k}(1+O(1 / k)+O(k / n)) \rho(n-k, n / 2+s-k) \\
& \times \frac{(n-k)^{2 s-k}}{(n+2 s-2 k)^{n / 2+s-k}} \frac{1}{k^{7 / 2} e^{k}} \exp \left(\frac{\gamma^{4 / 3} k}{2}\left(\frac{(n+2 s-2 k)}{(n-k)^{2}}\right)^{2 / 3}\right) .
\end{aligned}
$$

We shall sum over $k$ in (21) or, more specifically, over $r$ for $k=2 s+r$, where $r=$ $r(n, s)$ will shortly be determined. Letting $k=2 s+r$, we estimate the summands in (21) as

$$
\begin{aligned}
\frac{(n-k)^{2 s-k}}{(n+2 s-2 k)^{n / 2+s-k}} & =(n-2 s)^{-(n / 2-s)}\left(1-\frac{r}{n-2 s}\right)^{-r}\left(1-\frac{2 r}{n-2 s}\right)^{r-n / 2-s} \\
& \stackrel{\text { 1117 }}{=}(n-2 s)^{-(n / 2-s)} \exp \left(r-\frac{r^{3}}{6(n-2 s)^{2}}+O\left(\frac{r^{4}}{(n-2 s)^{3}}\right)\right) .
\end{aligned}
$$


Therefore (21) becomes

$$
\begin{aligned}
& \operatorname{pl}(n, n / 2+s)=(1+O(1 / n)+O(1 / s)+O(s / n)) \frac{g 3^{5 / 2}}{2^{7} \pi^{1 / 2} e^{3 / 4} \gamma^{10 / 3}} n^{n+7 / 6} e^{n / 2-s} \\
& \quad \times(n-2 s)^{-(n / 2-s)} \exp \left(\frac{\gamma^{4 / 3} s}{(n-2 s)^{2 / 3}}\right) \sum_{r} \rho(n-2 s-r, n / 2-s-r) \varphi(r),
\end{aligned}
$$

where the function $\varphi(r)=\varphi_{n, s}(r)$ is defined as

$$
\varphi(r):=(s+r / 2)^{-7 / 2} \exp \left(\frac{\gamma^{4 / 3} r}{2(n-2 s)^{2 / 3}}-\frac{r^{3}}{6(n-2 s)^{2}}+O\left(\frac{r^{4}}{(n-2 s)^{3}}\right)+O\left(\frac{r}{n}\right)\right) .
$$

Observe that the main contribution to $\varphi(r)$, and therefore to (22), comes from the term $r=O\left(n^{2 / 3}\right)$. Since $n / 2-s-r=(n-2 s-r) / 2-r / 2$, from Theorem 8 (ii) and the definition of (16) we have $\rho(n-2 s-r, n / 2-s-r) \rightarrow \nu(-x / 2)$, when $r /(n-2 s-r)^{2 / 3} \rightarrow x$. Thus, the sum over $r=O\left(n^{2 / 3}\right)$ in (22) can be replaced by an integral over $x=r n^{-2 / 3}$, and we get

$$
\begin{aligned}
& \sum_{r} \rho(n-2 s-r, n / 2-s-r) \varphi(r) \\
& \quad=(1+o(1)) n^{2 / 3} s^{-7 / 2} \int_{-\infty}^{\infty} \exp \left(-\frac{x^{3}}{6}+\frac{\gamma^{4 / 3} x}{2}\right) \nu\left(-\frac{x}{2}\right) d x .
\end{aligned}
$$

As a consequence, the first part of the theorem follows.

For the second part of the assertion note that in this case the main contribution to the sum (18) follows from $\ell$ 's close to $\ell_{0}$ given in (20) and $k=(2+o(1)) s$, and so a.a.s. $\operatorname{ex}(n, M)=\left(\frac{2 \gamma^{4 / 3}}{3}+o(1)\right) \frac{s}{n^{2 / 3}}$. Therefore, by Theorem 2, a.a.s. $P(n, M)$ is clean, its kernel has

$$
(2+o(1)) \operatorname{ex}(n, M)=\left(\frac{4 \gamma^{4 / 3}}{3}+o(1)\right) \frac{s}{n^{2 / 3}}
$$

vertices, and $\operatorname{cr}(n, M)=\left(2 \gamma^{2 / 3}+o(1)\right) \frac{s}{n^{1 / 3}}$.

5.6. Middle range. As far as $M / n$ is bounded away from both 0 and 1 we can prove results similar to Theorem [11, but since now $\ell=O\left(n^{1 / 3}\right)=O\left(k^{1 / 3}\right)$, we can estimate $\operatorname{pl}(n, M)$ only up to a constant factor.

Theorem 12. If $M=$ an for a constant $1 / 2<a<1$, then

$$
\operatorname{pl}(n, M)=\Theta(1) n^{a n-5 / 3}\left(\frac{e}{2-2 a}\right)^{n-a n} \exp \left(\gamma^{4 / 3}(a-1 / 2) n^{1 / 3}\right) .
$$

Furthermore, a.a.s. $L_{1}(n, M)=(2 a-1+o(1)) n$, $\operatorname{ex}(n, M)=\Theta\left(n^{1 / 3}\right)$, and $\operatorname{cr}(n, M)$ $=\Theta\left(n^{2 / 3}\right)$.

Proof. Following along the lines of the proof of Theorem 11, but with $s$ replaced by an $-n / 2$, yields the assertion.

Using Theorems 9.12 one can find that the threshold for the property that $P(n, M)$ has the chromatic number four is $M=n+o(n)$.

Theorem 13. Let $\epsilon>0$.

(i) If $M \leq(1-\epsilon) n$, then a.a.s. $\chi(P(n, M))=3$. 
(ii) If $M \geq(1+\epsilon) n$, then a.a.s. $P(n, M)$ contains a copy of $K_{4}$ and, as a consequence, $\chi(P(n, M))=4$.

Proof. Here we only sketch the argument. Let $M=a n, 1 / 2<a<1$. Then a.a.s. the kernel of $P(n, M)$ has deficiency $\Theta(1)$ and $\Theta\left(n^{1 / 3}\right)$ vertices (see Theorems 2 and 12). Furthermore, on the edges of the kernel we need to place $\Theta\left(n^{2 / 3}\right)$ vertices of the core. Thus, the probability that on some edge we place fewer than five vertices is $\Theta\left(n^{-1 / 3}\right)$, and so there are a.a.s. at most $\ln \ln n$ edges of the kernel of $P(n, M)$ which contain fewer than three vertices of the core. Moreover, none of the vertices of the kernel is incident with more than one such edge. It is easy to see that such a graph can be colored using three colors. A similar argument shows that $\chi(P(n, M)) \leq 3$ for $M \leq n / 2+o(n)$. On the other hand, from the formula for $\operatorname{pl}(n, M)$ for $M \geq(1+\epsilon) n$ by Giménez and Noy [16] and Chebyshev's inequality it follows that for such an $M$ the graph $P(n, M)$ contains a copy of $K_{4}$ (in fact it a.a.s. contains $\Theta(n)$ copies of $K_{4}$, in which three vertices have degree three in $P(n, M))$.

Let us remark that Dowden [10] studied the probability that $P(n, M)$ contains a given subgraph and determined its asymptotic behavior depending on the ratio $M / n$. In particular, Theorem 13 (ii) is relevant to his Theorem 17 .

5.7. Second critical range. In the previous section we showed that as far as $M=$ an and $a \in(1 / 2,1)$, the size of the largest component grows with $M$, but its density does not depend much on the value of $a$ and in the whole range is of the order $n^{1 / 3}$. Clearly, this situation must change when the size of the largest component is $n-o(n)$. Indeed, starting from some point, the increase in the number of edges of $P(n, M)$ must contribute to the density of the largest component, since when $M=n+t$, for $t$ large enough, we should expect $\operatorname{ex}(n, M)=(1+o(1)) t$. Our next result states that this change occurs when $M=n+O\left(n^{3 / 5}\right)$.

Theorem 14. Set $M=n+t$, where $t=o(n)$.

(i) Let $w=w(n, t)=\frac{\gamma^{4 / 3}(n-2|t|)}{3 \cdot 2^{2 / 3}|t|^{2 / 3}}$. If $t \ll-n^{3 / 5}$, but $n / 2+t \gg n^{2 / 3}$, then

$$
\begin{aligned}
\operatorname{pl}(n, M)=\Theta(1) & n^{n-1 / 2} \frac{(2|t|+2 w)^{t+1 / 6}}{(3|t|+5 w)^{1 / 2} w^{5 / 2}}\left(\frac{|t|}{|t|+w}\right)^{w} \\
& \times \exp \left(\frac{5 w}{2}+|t|-\frac{3 w^{2}}{n-2|t|}+\beta \cdot \frac{\gamma^{2}}{3^{3 / 2} 2} \frac{n-2|t|}{|t|}\right) .
\end{aligned}
$$

Furthermore, a.a.s. $L_{1}(n, M)=n-(2+o(1))|t| \operatorname{ex}_{\mathrm{c}}(n, M)=(1+o(1)) w$, and $\operatorname{cr}_{\mathrm{c}}(n, M)=\left(\frac{\gamma^{2 / 3}}{2^{1 / 3}}+o(1)\right) \frac{n-2|t|}{|t|^{1 / 3}}$.

(ii) Let $b$ be the unique positive solution of the equation $b^{3 / 2}(b-c)=\frac{\gamma^{2}}{2 \cdot 3^{3 / 2}}$. If $t=c \cdot n^{3 / 5}$ for $c \in(-\infty, \infty)$, then

$$
\begin{aligned}
\operatorname{pl}(n, M)=\Theta(1) & n^{n-1 / 2} t^{-17 / 6}(2(b / c-1) t)^{t} \\
& \times \exp \left(\left(\frac{5 b}{2 c}-1\right) t-\frac{3 b}{c}\left(\frac{b}{c}-1\right) \frac{t^{2}}{n}+\beta \cdot \frac{(b t / c)^{3 / 2}}{n^{1 / 2}}\right) .
\end{aligned}
$$

Furthermore, a.a.s. $L_{1}(n, M)=n-(2 b-2 c+o(1)) n^{3 / 5}, \operatorname{ex}_{\mathrm{c}}(n, M)=$ $(b+o(1)) n^{3 / 5}$, and $\mathrm{cr}_{\mathrm{c}}(n, M)=\Theta\left(n^{4 / 5}\right)$. 
(iii) Let $z=z(n, t)=\frac{\gamma^{2}}{2 \cdot 3^{3 / 2}}\left(\frac{n}{t}\right)^{3 / 2}$. If $t \gg n^{3 / 5}$, then

$$
\begin{aligned}
\operatorname{pl}(n, M)=\Theta(1) & \frac{n^{n-1 / 2}(2 z)^{t+1 / 6}}{(2 t+5 z)^{1 / 2}(t+z)^{5 / 2}}\left(\frac{t}{t+z}\right)^{3(t+z) / 2} \\
& \times \exp \left(\frac{3}{2} t+\frac{5}{2} z-\frac{3 z(t+z)}{n}+\beta \cdot \frac{t^{3 / 2}}{n^{1 / 2}}\right) .
\end{aligned}
$$

Furthermore, if in addition $t \ll n^{2 / 3}$, a.a.s. $L_{1}(n, M)=n-\left(\gamma^{2} / 3^{3 / 2}+\right.$ $o(1))(n / t)^{3 / 2}, \operatorname{ex}_{\mathrm{c}}(n, M)=\left(1+o(1)(t+z)\right.$, and $\mathrm{cr}_{\mathrm{c}}(n, M)=\Theta(\sqrt{n t})$.

Proof. In order to show (i) we follow the argument presented in Section 5.5. Thus, from Theorem 2 and Theorem 8 , we get

$$
\begin{aligned}
\operatorname{pl}(n, n+t) & =\sum_{k, \ell}\left(\begin{array}{l}
n \\
k
\end{array}\right) C(n-k, n-k+\ell) U(k, k+t-\ell) \\
& =(1+o(1)) \frac{g 3^{1 / 2}}{\pi 2^{5}} n^{n-1 / 2} \sum_{\ell}\left(\frac{\gamma^{2} e^{3 / 2}}{3^{3 / 2}}\right)^{\ell} \frac{1}{\ell^{3 \ell / 2+3}} \sum_{k} \psi(k),
\end{aligned}
$$

where the function $\psi(k)=\psi_{n, t}(k)$ is defined as

$$
\begin{gathered}
\psi(k):=\rho(k, k+t-\ell)(k(k+t-\ell))^{-1 / 2}(n-k)^{3 \ell / 2} k^{k+2 t-2 \ell}\left(\frac{e}{2(k+t-\ell)}\right)^{k+t-\ell} \\
\times \exp \left(-\frac{k+t-\ell}{k}-\left(\frac{k+t-\ell}{k}\right)^{2}+O\left(\frac{1}{k}\right)\right. \\
\left.+O\left(\frac{k}{n}\right)+\beta \sqrt{\frac{\ell^{3}}{n-k}}+O\left(\frac{\ell^{2}}{n-k}\right)\right) .
\end{gathered}
$$

In order to estimate (26) observe that the maximum of the sum $\sum_{k} \psi(k)$ is taken at $k_{0}=2(\ell-t)$. More precisely, the main contribution to the sum comes from the terms $k=2(\ell-t)+r$, where $r=O\left((2(\ell-t))^{2 / 3}\right)$. Thus,

$$
\begin{aligned}
\sum_{k} \psi(k)=(1 & +o(1)) e^{-3 / 4} 2^{1 / 2} \frac{e^{\ell-t}(n-2(\ell-t))^{3 \ell / 2}}{(2(\ell-t))^{\ell-t+1}} \\
& \times \sum_{r} \rho(2(\ell-t)+r, \ell-t+r) \exp \left(\frac{r^{3}}{6(2(\ell-t))^{2}}-\frac{3 r \ell}{2(n-2(\ell-t))}\right) \\
& \times \exp \left(\beta \sqrt{\frac{\ell^{3}}{n-2(\ell-t)}}+O\left(\frac{\ell^{2}}{n-2(\ell-t)}\right)\right),
\end{aligned}
$$

where we sum over $r=O\left((2(\ell-t))^{2 / 3}\right)$. Note also that $\ell-t+r=\frac{2(\ell-t)+r}{2}+\frac{r}{2}$, and so if $r /(2(\ell-t)+r)^{2 / 3} \rightarrow x$, then $\rho(2(\ell-t)+r, \ell-t+r) \rightarrow \nu\left(\frac{x}{2}\right)$. 
Next note that the main contribution to the sum over $\ell$ in (26) comes from the terms $\ell=\ell_{0}+O\left(\sqrt{\ell_{0}}\right)$, where $\ell_{0}=\frac{\gamma^{4 / 3}\left(n-2\left(\ell_{0}-t\right)\right)}{3\left(2\left(\ell_{0}-t\right)\right)^{2 / 3}}$, so (26) can be estimated by (27)

$$
\begin{aligned}
\operatorname{pl}(n, n+t)=(1 & +o(1)) \frac{g 3^{1 / 2}}{\pi 2^{9 / 2} e^{3 / 4}} n^{n-1 / 2} \int_{-\infty}^{\infty} \exp \left(\frac{x^{3}}{6}-\frac{\gamma^{4 / 3} x}{2}\right) \nu\left(\frac{x}{2}\right) d x \\
& \times A(n, t) \exp \left(\beta \sqrt{\frac{\ell_{0}^{3}}{n-2\left(\ell_{0}-t\right)}}+O\left(\frac{\ell_{0}^{2}}{n-2\left(\ell_{0}-t\right)}\right)\right)
\end{aligned}
$$

where the function $A(n, t)$ is defined as

$$
A(n, t):=\sum_{\ell}\left(\frac{\gamma^{2} e^{3 / 2}}{3^{3 / 2}}\right)^{\ell} \frac{e^{\ell-t}(n-2(\ell-t))^{3 \ell / 2}}{\ell^{3 \ell / 2+3}(2(\ell-t))^{\ell-t+1 / 3}} .
$$

The behavior of $A(n, t)$ depends on the range of $t$. Thus, we consider three cases, corresponding to the periods described in the three parts of the assertion of Theorem 14,

First, let $t \ll-n^{3 / 5}$, but $n / 2+t \gg n^{2 / 3}$. The main contribution to the sum $A(n, t)$ comes from the terms $\ell=\ell_{0}+O\left(\sqrt{\ell_{0}}\right)$ with $\ell_{0}=w$, where

$$
w=w(n, t):=\frac{\gamma^{4 / 3}(n-2|t|)}{3 \cdot|2 t|^{2 / 3}} .
$$

In this case we have

$$
A(n, t)=(1+o(1))(2 \pi)^{1 / 2} \frac{(2(w-t))^{t+1 / 6}}{(5 w-3 t)^{1 / 2} w^{5 / 2}}\left(\frac{|t|}{w-t}\right)^{w} \exp \left(\frac{5 w}{2}-t-\frac{3 w^{2}}{n+2 t}\right),
$$

and (23) follows. In order to get the information on the structure of $P(n, M)$ for this range, one should apply Theorem 2 and observe that

$$
\sqrt{\frac{\ell_{0}^{3}}{n-2\left(\ell_{0}-t\right)}}=(1+o(1)) \frac{\gamma^{2}}{3^{3 / 2} 2} \frac{n+2 t}{|t|} .
$$

Now let $t=c n^{3 / 5}$ for some constant $c \in(-\infty, \infty)$. In this case, the main contribution to the sum $A(n, t)$ comes from the terms $\ell=\ell_{0}+O\left(\sqrt{\ell_{0}}\right)$ with $\ell_{0}=$ $b t / c$, where $b$ is the unique positive solution of the equation $b^{3 / 2}(b-c)=\frac{\gamma^{2}}{2 \cdot 3^{3 / 2}}$. We have

$$
\begin{aligned}
A(n, t)=(1+o(1))(2 \pi)^{1 / 2} & \frac{(2(b-c))^{1 / 6} c^{3}}{(5 b-3 c)^{1 / 2} b^{5 / 2}} t^{-17 / 6}(2(b / c-1) t)^{t} \\
& \times \exp \left(\left(\frac{5 b}{2 c}-1\right) t-\frac{3 b}{c}\left(\frac{b}{c}-1\right) \frac{t^{2}}{n}\right)
\end{aligned}
$$

and

$$
\sqrt{\frac{\ell_{0}^{3}}{n-2\left(\ell_{0}-t\right)}}=(1+o(1)) \frac{(b t / c)^{3 / 2}}{n^{1 / 2}} .
$$

Therefore, (ii) follows from (27) and Theorem 2 ,

Finally, let $t \gg n^{3 / 5}$ but $t=o(n)$. Then the main contribution to the sum $A(n, t)$ comes from the terms $\ell=\ell_{0}+O\left(\sqrt{\ell_{0}}\right)$ with $\ell_{0}=t+z$, where

$$
z=z(n, t):=\frac{\gamma^{2}}{2 \cdot 3^{3 / 2}}\left(\frac{n}{t}\right)^{3 / 2}
$$


We have

$$
\begin{aligned}
A(n, t)=(1+o(1))(2 \pi)^{1 / 2} & \frac{(2 z)^{t+1 / 6}}{(2 t+5 z)^{1 / 2}(t+z)^{5 / 2}}\left(\frac{t}{t+z}\right)^{3(t+z) / 2} \\
& \times \exp \left(\frac{3}{2} t+\frac{5}{2} z-\frac{3 z(t+z)}{n}\right)
\end{aligned}
$$

which, together with (27), gives (25).

The second part of (iii) follows from (27), Theorem 2, and the observation that

$$
\sqrt{\frac{\ell_{0}^{3}}{n-2\left(\ell_{0}-t\right)}}=(1+o(1)) \frac{t^{3 / 2}}{n^{1 / 2}} .
$$

We also remark that we expect the estimates for $\operatorname{ex}_{\mathrm{c}}(n, M)$ and $\mathrm{cr}_{\mathrm{c}}(n, M)$ to also hold for $n^{2 / 3} \leq t \ll n$, but the error term $O\left(t^{3 / 2} n^{-1 / 2}\right)$ in (25) becomes too large to provide precise information on the structure of $P(n, M)$. Let us also point out that if the Giant Conjecture is true, then $\operatorname{ex}(n, M)=(1+o(1)) \operatorname{ex}_{\mathrm{c}}(n, M)$ and $\operatorname{cr}(n, M)=(1+o(1)) \mathrm{cr}_{\mathrm{c}}(n, M)$ in the whole range of $M$.

\section{Concluding Remarks}

Let us first propose a heuristic which explains the behavior of $P(n, M)$ as described in Theorems 914. Note that the number $\operatorname{pl}(n, M)$ of planar graphs with $n$ vertices and $M$ edges can be computed in two different ways. Our estimates were based on the formula

$$
\operatorname{pl}(n, M)=\sum_{k, \ell}\left(\begin{array}{l}
n \\
k
\end{array}\right) C(k, k+\ell) U(n-k, M-k-\ell),
$$

where we extracted from the graph its complex part. But one could also use the formula

$$
\operatorname{pl}(n, M)=\sum_{k, \ell}\left(\begin{array}{l}
n \\
k
\end{array}\right) C^{c o n n}(k, k+\ell) R_{p l}(n-k, M-k-\ell),
$$

where we first identify in the graph the largest component of $k$ vertices and $k+\ell$ edges, which typically is complex and unique, and then we supplement it by a random planar graph of $n-k$ vertices and $M-k-\ell$ edges. However, Theorem 2 (iv) states that (at least for small $\ell$ ) a graph chosen at random from all complex planar graphs with $k$ vertices and $k+\ell$ edges consists of the giant component of size $k-O(k / \ell)$ and, possibly, some small components of finite complexity and size $\Theta(k / \ell)$. If the Giant Conjecture is true, it is in fact the case for all values of $\ell \leq k$. Thus, the planar graph which is outside the largest component must contain just a few (if any) components which are complex. It happens only if its density is such as the density of the standard uniform graph model in the critical period. Consequently, in (28), we must have

$$
M-k-\ell=(n-k) / 2+\Theta\left((n-k)^{2 / 3}\right),
$$

and, since in the critical period the sizes of all complex components of a random graph on $n$ vertices are of the order $n^{2 / 3}$,

$$
k / \ell=\Theta\left((n-k)^{2 / 3}\right) .
$$


Let us check what it gives when $M=n / 2+s$ with $s \gg n^{2 / 3}$ to ensure that $P(n, M)$ contains a complex and unique giant component. If $k \ll M$, then from (29) we get

$$
L_{1}(n, M)=2 M-n+O(\ell)+O\left(n^{2 / 3}\right)=2 s+O(\ell)+O\left(n^{2 / 3}\right),
$$

and, by (30),

$$
\ell=\Theta\left(\frac{k}{(n-k)^{2 / 3}}\right)=\Theta\left(\frac{s}{(n-s)^{2 / 3}}\right),
$$

which fits perfectly the estimates from Theorems 11 and 12 . Now let $M=n-t$, $t=o(n)$. Then, as before, from (29) and (30) we infer that

$$
n-L_{1}(n, M)=2 t+O\left(\left(n-L_{1}(n, M)\right)^{2 / 3}\right)+O(\ell)
$$

and

$$
\ell=\Theta\left(\frac{n}{\left(n-L_{1}(n, M)\right)^{2 / 3}}\right) .
$$

Note that when $-t$ is large, then, clearly, $\ell=\Theta(|t|)$, and the error term $O(\ell)$ in (31) is equal to the main term $2 t$. In order to compute this 'threshold value' of $t$ one should use (32) and substitute to it $\ell=\Theta(|t|)$ and $n-L_{1}(n, M)=\Theta(t)$. It gives $|t|=\Theta\left(n^{3 / 5}\right)$ as the threshold value for the property that $\ell=\Theta(|t|)$. Consequently, if $t \gg n^{3 / 5}$, then we have $n-L_{1}(n, M)=(2+o(1)) t$, as stated in Theorem 14 (i), while for $t \ll-n^{3 / 5}$, we have $\ell=\Theta(|t|)$ and, by (32), $n-L_{1}(n, M)=\Theta\left((n / t)^{3 / 2}\right)$, which agrees with Theorem 14 (iii).

Since for $M<a n$, where $a<1$, the random graph $P(n, M)$ is a.a.s. quite sparse (see Theorem 12), while for $M>a n, a>1$, it is quite dense (e.g. a.a.s. it contains a copy of any given planar graph), it seems that the most interesting period in the evolution of $P(n, M)$ is for $M=n-o(n)$. This intuition is confirmed by Theorem 13, but clearly much remains to be done. For instance, it seems that the correct threshold function for the property that $\chi(P(n, M))=4$ is $M=n+\Theta\left(n^{7 / 9}\right)$, more precisely, we conjecture that the following holds.

Conjecture. If $(M-n) / n^{7 / 9} \rightarrow 0$, then a.a.s. $\chi(P(n, M)) \leq 3$, while if $(M-$ $n) / n^{7 / 9} \rightarrow \infty$, then a.a.s. $P(n, M) \supseteq K_{4}$ and so $\chi(P(n, M))=4$.

Let us briefly justify the above claim that $K_{4}$ emerges in $P(n, M)$ when $M=$ $n+\Theta\left(n^{7 / 9}\right)$. First of all, in order to have a single copy of $K_{4}$ in $P(n, M)$ we need many copies of $K_{4}$ in the kernel of the largest component of $P(n, M)$. The number of copies of $K_{4}$ in the kernel should be of the same order as the number of vertices of degree four in the kernel, which, in turn, is expected to be of the order of deficiency of the graph. Once we have a copy of $K_{4}$ in the kernel, the probability that after placing vertices of the core at the edges of the kernel none of these vertices will be put at one of six edges of $K_{4}$ is $\Theta\left((\operatorname{ker}(n, M) / \operatorname{core}(n, M))^{6}\right)$. Hence, if our estimates for $\operatorname{ker}(n, M)$ and $\operatorname{core}(n, M)$ from Theorem 14 (iii) remain valid for all values of $M$ such that $n^{3 / 5} \ll M-n \ll n$, then the expected number of copies of $K_{4}$ 's in $P(n, M)$ is of the order

$$
\operatorname{df}(n, M) \cdot\left(\frac{\operatorname{ker}(n, M)}{\operatorname{core}(n, M)}\right)^{6} \sim \sqrt{\frac{\ell^{3}}{k}}\left(\frac{\ell}{\sqrt{k \ell}}\right)^{6}=\ell^{9 / 2} k^{-7 / 2} \sim t^{9 / 2} n^{-7 / 2} .
$$

Thus, this number is bounded away from zero for $t=\Theta\left(n^{7 / 9}\right)$. 
Let us also add a few words on the models of random planar graphs different from $P(n, M)$. One of the most natural is the graph obtained by the random planar process, when we add to an empty graph on $n$ vertices $M$ edges one by one, each time choosing a new edge uniformly at random from all pairs which preserve planarity of the graph (see Gerke et al. 15). In this model the structure of components is similar to that of a standard graph $G\left(n, M^{\prime}\right)$ for an appropriately chosen $M^{\prime} \geq M$. Another model of a random planar graph is the binomial random graph $P(n, p)$, when we look at properties of $G(n, p)$ conditioned on the fact that it is planar. Equivalently, one can view $P(n, p)$ as the graph chosen from the family $\mathcal{P}(n)$ of all planar graphs on $n$ vertices in such a way that each $G \in \mathcal{P}(n)$ appears as $P(n, p)$ with the probability

$$
\mathbb{P}(P(n, p)=G)=p^{e(G)}(1-p)^{\left(\begin{array}{l}
n \\
2
\end{array}\right)-e(G)} / Z(n, p),
$$

where $e(G)$ denotes the number of edges of $G$, and

$$
Z(n, p)=\sum_{G \in \mathcal{P}(n)} p^{e(G)}(1-p)^{\left(\begin{array}{l}
n \\
2
\end{array}\right)-e(G)} .
$$

Since clearly for every property $\mathcal{A}$

$$
\mathbb{P}(P(n, p) \text { has } \mathcal{A} \mid e(P(n, p))=M)=\mathbb{P}(P(n, M) \text { has } \mathcal{A}),
$$

once we determine the typical number of edges in $P(n, p)$ the problem of finding properties of $P(n, p)$ reduces to studying these properties for $P(n, M)$. From the estimates of $\mathrm{pl}(n, M)$ given in Theorems 9012 and 14, it follows that if $n p \leq 1$, then a.a.s. $P(n, p)$ has $M=(1+o(1)) p\left(\begin{array}{l}n \\ 2\end{array}\right)$ edges; if $1 / n \leq p \ll n^{-3 / 5}$, then a.a.s. $M=n-(1+o(1)) /(2 p)$; for $p=O\left(n^{-3 / 5}\right)$ we are in the second critical period, i.e. a.a.s. $M=n+O\left(n^{3 / 5}\right)$; finally for $p \gg n^{-3 / 5}$ we have a.a.s. $M=n+\Theta\left(p^{2 / 3} n\right)$. Note that a large part of the evolution of $P(n, p)$, when $1 / n \ll p \ll 1$, corresponds to the period of evolution of $P(n, M)$ when $M=n+o(n)$, which, as we have already remarked, is crucial for many properties of $P(n, M)$.

Another interesting model is a random cluster model $P(n, M, q)$ on planar graphs when for every labeled planar graph with vertex set $[n]$ we put

$$
\mathbb{P}(P(n, M, q)=G)=q^{c(G)} / Z(n, M, q),
$$

where $q>1$ is a parameter, $c(G)$ stands for the number of components in $G$, and $Z(n, M, q)$ is the normalizing factor. In a similar way one can define $P(n, p, q)$ by adding factors $q^{c(G)}$ to the right hand sides of (33) and (34). It is well known that the additional cluster factor $q^{c(G)}$ in, say, the standard model $G(n, p)$ leads to an interesting phenomena such as the discontinuous phase transition which occurs in $G(n, p, q)$ for $q>2$ (cf. Luczak and Luczak 26). Unfortunately, no such event can be observed in the planar case. The evolution of $P(n, M, q)$ is quite similar to that of $P(n, M)=P(n, M, 1)$. The reason is quite simple: the giant complex component of $P(n, M)$ is very sparse until it reaches the size $n-o(n)$, and so the number of components is always close to $n-M+o(n)$ and, as calculations show, cannot be influenced much by the presence of the additional factor $q^{c(G)}$. The asymptotic behavior of $P(n, p, q)$ does not depend very much on the value of $q$ either, except for the scaling: the number of edges of $P(n, p, q)$ is roughly the same as for $P(n, p / q)$. 


\section{ACKNOWLEDGMENTS}

A part of this work was carried out when both the authors visited the MittagLeffler Institute and was completed during the first author's stay at the Adam Mickiewicz University within the DFG Heisenberg Programme. We would like to thank these institutions for their support. The second author was supported by the Foundation for Polish Science.

\section{REFERENCES}

[1] C. Banderier, P. Flajolet, G. Schaeffer, and M. Soria, Random maps, coalescing saddles, singularity analysis, and Airy phenomena, Random Struc. Alg. 19 (2001), 194-246. MR 1871555 (2002k:05012)

[2] E. A. Bender and E. R. Canfield, The number of rooted maps on an orientable surface, J. Combin. Theory Ser. B 53 (1991), 293-299. MR1129556 (92g:05100)

[3] A. Bender, Z. Gao, and N. C. Wormald, The number of labeled 2-connected planar graphs, Electron. J. Combin. 9 (2002), Research Paper 43, 13 pp. MR1946145 (2003i:05071)

[4] M. Bodirsky, M. Kang, M. Löffler, and C. McDiarmid, Random cubic planar graphs, Random Struc. Alg. 30 (2007), 78-94. MR2283223 (2007k:05193)

[5] B. Bollobás, The evolution of random graphs, Trans. Am. Math. Soc. 286 (1984), 257-274. MR756039 (85k:05090)

[6] J. Bouttier, P. Di Francesco, and E. Guitter, Planar maps as labeled mobiles, Electron. J. Combin. 11 (2004), Research Paper 69, 27 pp. MR2097335 (2005i:05087)

[7] V. E. Britikov, Asymptotics of the number of forests made up of nonrooted trees, Math. Notes 43 (1988), 387-394. MR.954351 (89j:05063)

[8] G. Chapuy, É. Fusy, O. Giménez, B. Mohar, and M. Noy, Asymptotic enumeration and limit laws for graphs of fixed genus, to appear in SIAM Journal of Discrete Mathematics.

[9] P. Chassaing and G. Schaeffer, Random planar lattices and integrated superBrownian excursion, Probab. Theory Related Fields 128 (2004), 161-212. MR2031225 (2004k:60016)

[10] C. Dowden, The evolution of uniform random planar graphs, Electron. J. Combin. 17 (2010), Research Paper 7. MR2578902 (2011c:05306)

[11] P. Erdős and A. Rényi, On the evolution of random graphs, Publ. Math. Inst. Hungar. Acad. Sci. 5 (1960), 17-61. MR0125031(23:A2338)

[12] A. Frieze, Random Graphs '85: Open problems, Annals of Discrete Mathematics 33 (1987), 353-354. MR.930478(88j:05002)

[13] J. F. Le Gall, The topological structure of scaling limits of large planar maps, Invent. Math. 169 (2007), 621-670. MR 2336042 (2008i:60022)

[14] S. Gerke, C. McDiarmid, A. Steger, and A. Weißl, Random planar graphs with $n$ nodes and a fixed number of edges, In Proceedings of the Sixteenth Annual ACM-SIAM Symposium on Discrete Algorithms (SODA'05), pages 999-1007, 2005. MR2298359

[15] S. Gerke, D. Schlatter, A. Steger, and A. Taraz, The random planar graphs process, Random Struc. Alg. 32 (2008), 236-261. MR2387559 (2008m:60015)

[16] O. Giménez and M. Noy, Asymptotic enumeration and limit laws of planar graphs, J. Amer. Math. Soc. 22 (2009), 309-329. MR2476775 (2010g:05031)

[17] F. Harary and E. Palmer, Graphical enumeration, Academic Press, New York-London, 1973. MR0357214(50:9682)

[18] S. Janson, The growth of components in random graphs, Random Struc. Alg. 17 (2000), 343-356. MR.1801138(2002j:05133)

[19] S. Janson, D. Knuth, T. Łuczak, and B. Pittel, The birth of the giant component, Random Struc. Alg. 4 (1993), 233-358. MR1220220 (94h:05070)

[20] S. Janson, T. Łuczak, and A. Ruciński, Random Graphs, Wiley, New York, 2000. MR1782847 (2001k:05180)

[21] T. Łuczak, Component behavior near the critical point of the random graph, Random Struc. Alg. 1 (1990), 287-310. MR.1099794 (92c:05139)

[22] T. Euczak, Cycles in random graphs, Discrete Math. 98 (1991), 231-236. MR.1144405 (92i:05190) 
[23] T. Euczak, The phase transition in a random graph, In Combinatorics, Paul Erdös is eighty, Vol. 2, Bolyai Soc. Math. Stud., 2, J. Bolyai Math. Soc., Budapest, 1996, 399-422. MR:1395867 (97d:05245)

[24] T. Łuczak and B. Pittel, Components of random forests, Comb. Probab. Comput. 1 (1992), 35-52. MR 1167294 (93i:05118)

[25] T. Łuczak, B. Pittel, and J. Wierman, The structure of a random graph near the point of the phase transition, Trans. Amer. Math. Soc. 341 (1994), 721-748. MR.1138950 (94d:05123)

[26] M. J. Luczak and T. Euczak, The phase transition in the cluster-scaled model of a random graph, Random Struc. Alg. 28 (2006), 215-246. MR2198498(2006k:05193)

[27] C. McDiarmid, Random graphs on surfaces, J. Combin. Theory Ser. B 98 (2008), 778-797. MR2418771 (2009c:05224)

[28] C. McDiarmid and B. Reed, On the maximum degree of a random planar graph, Combin. Probab. Comput. 17 (2008), 591-601. MR2433943(2009m:05165)

[29] C. McDiarmid, A. Steger, and D. Welsh, Random planar graphs, J. Combin. Theory Ser. B 93 (2005), 187-205. MR2117936 (2005k:05221)

[30] D. Osthus, H. J. Prömel, and A. Taraz, On random planar graphs, the number of planar graphs and their triangulations. J. Combin. Theory Ser. B 88 (2003), 119-134. MR 1973264 (2004a:05068)

[31] G. Schaeffer, Conjugaison d'arbres et cartes combinatoires aléatoires, Ph.D. Thesis (in French), Université Bordeaux I, 1998.

[32] O. Schramm, Conformally invariant scaling limits: An overview and a collection of problems. In International Congress of Mathematicians. Vol. I, pages 513-543. Eur. Math. Soc., Zürich, 2007. MR2334202 (2008j:60237)

[33] W. T. Tutte, A census of planar maps, Canad. J. Math. 15 (1963), 249-271. MR0146823 $(26: 4343)$

[34] W. T. Tutte, A census of planar triangulations, Canad. J. Math. 14 (1962), 21-38. MR0130841 (24:A695)

Institut für Optimierung und Diskrete Mathematik (Math B), Technische UniverSität Graz, Steyrergasse 30, A-8010 Graz, Austria

E-mail address: kang@math.tugraz.at

Department of Discrete Mathematics, Adam Mickiewicz University, 61-614 Poznań, POLAND

E-mail address: tomasz@amu.edu.pl 